\title{
Decay Rates of the Full Compressible Hall-MHD Equations for Quantum Plasmas
}

\section{Yu Gao}

College of Science, University of Shanghai for Science and Technology, Shanghai, China

Email: gaoyu15762185220@163.com

How to cite this paper: Gao, Y. (2019) Decay Rates of the Full Compressible HallMHD Equations for Quantum Plasmas. Journal of Applied Mathematics and Physics, 7, 2603-2631.

https://doi.org/10.4236/jamp.2019.711178

Received: September 23, 2019

Accepted: November 2, 2019

Published: November 5, 2019

Copyright () 2019 by author(s) and Scientific Research Publishing Inc. This work is licensed under the Creative Commons Attribution International License (CC BY 4.0).

http://creativecommons.org/licenses/by/4.0/

\section{Open Access}

\begin{abstract}
In this paper, we are concerned with the Cauchy problem of the full compressible Hall-magnetohydrodynamic equations in three-dimensional whole space. By the energy method, global existence of a unique strong solution is established. If further that the $L^{1}$-norm of the perturbation is bounded, we prove the decay rates in time of the solution and its first-order derivatives in $L^{2}$-norm via some $L^{p}-L^{q}$ estimates by the linearized operator.
\end{abstract}

\section{Keywords}

Full Compressible Hall-Magnetohydrodynamic Equations, Global Existence, Decay Rates

\section{Introduction}

We consider with the Cauchy problem of the full compressible

Hall-magnetohydrodynamic (in short, Hall-MHD) equations in the whole space:

$$
\left\{\begin{array}{l}
\rho_{t}+\operatorname{div}(\rho u)=0, \\
(\rho u)_{t}+\operatorname{div}(\rho u \otimes u)+\nabla P(\rho, \theta)-\frac{\hbar^{2}}{2} \rho \nabla\left(\frac{\Delta \sqrt{\rho}}{\sqrt{\rho}}\right)=B \cdot \nabla B+\mu \Delta u+(\lambda+\mu) \nabla \nabla \cdot u, \\
C_{v}\left[(\rho \theta)_{t}+\operatorname{div}(\rho u \theta)\right]-\kappa \Delta \theta+\theta P_{\theta}(\rho, \theta) \nabla \cdot u=2 \mu|D(u)|^{2}+\lambda(\operatorname{divu})^{2}+|\nabla \times B|^{2}, \\
B_{t}+u \cdot \nabla B+\nabla \times((\nabla \times B) \times B)-\Delta B=B \cdot \nabla u, \quad \nabla \cdot B=0,
\end{array}\right.
$$

for $(t, x) \in[0,+\infty) \times \mathbb{R}^{3}$ with the initial data:

$$
\left.(\rho, u, \theta, B)\right|_{t=0}=\left(\rho_{0}(x), u_{0}(x), \theta_{0}(x), B_{0}(x)\right) .
$$

here $\rho>0, u=\left(u^{1}, u^{2}, u^{3}\right), \theta$ and $B=\left(B^{1}, B^{2}, B^{3}\right)$ represent the density, velocity, absolute temperature and magnetic field, respectively. $D(u)$ is the deformation tensor given by 


$$
D(u)=\frac{1}{2}\left[\nabla u+(\nabla u)^{t r}\right] .
$$

The smooth function $P=P(\rho, \theta)$ is the pressure satisfying $P_{\rho}(\rho, \theta)>0$ and $P_{\theta}(\rho, \theta)>0$ and for all $\rho>0, \theta>0 . \kappa>0$ is the coefficient of heat conduction. $C_{v}$ is the specific heat at constant volume. For $\mu$ and $\lambda$ are the first and second viscosity coefficients satisfying the usual physical condition

$$
\mu>0, \quad 2 \mu+3 \lambda \geq 0 .
$$

And $\hbar>0$ is the Planck constant. The $\operatorname{div}()$ represents the divergence and the symbol $\otimes$ denotes the kronecker multiplication such that

$$
u \otimes u=\left(u_{i} u_{j}\right), 1 \leq i, j \leq 3 .
$$

In the last couple of decades, the magneto-hydrodynamic equations and associated models with quantum effects are widely studied. According to the quantum correction, Wigner [1] first derived the quantum correction to the energy density for thermodynamic equilibrium, and the quantum correction term goes hand in hand with Bohm potential [2] [3]. The $\hbar^{2}$-term was advanced in [4]. People might refer to Haas [5] for more physical interpretations of the model. $\mathrm{Pu}$ et al. [6] recently got global existence of classical solutions for the full compressible quantum Navier-Stokes. Global existence and decay rate of smooth solutions to the constant profile is considered by $\mathrm{Pu}$ and $\mathrm{Xu}$ [7]. The system (1) is itself interesting because the energy equation also includes the quantum effects through the energy density $\rho$, which gives the system new features. This makes it differ in the previous given results.

If there is no quantum corrections (i.e. $\hbar=0$ ), this system reduces to the usual compressible Hall-MHD equations. Hall-MHD is needed in many current physics problems. The Hall-MHD is indeed necessary to solve problems, for example, magnetic reconnection in space plasma (see [8] [9]), formation and evolution of stars and neutron stars [10] [11] [12] and geo-dynamo [13] (see [14] for a detailed description of these physical processes). In contrast to the general MHD equations, the Hall-MHD equations have the Hall term $\nabla \times((\nabla \times B) \times B)$, which plays a significant role in magnetic reconnection. However, as far as we know, few achievements have been made in the study of the dynamics of global solutions to the 3D compressible Hall-MHD system, especially on the temporal decay of solutions. Very recently, global existence of smooth solutions to the $3 \mathrm{D}$ compressible Hall-MHD equations was first proved by Fan et al. [15], where the small initial disturbance belongs to $H^{3}\left(\mathbb{R}^{3}\right) \cap L^{1}\left(\mathbb{R}^{3}\right)$. More precisely, optimal time decay rate was also established. Later, the result from [15] was improved by Gao and Yao [16]. They obtained the global existence of strong solutions with the initial data are obtained in the lower regular spaces $H^{2}\left(\mathbb{R}_{x}^{3}\right)$ and proved optimal decay rates for the constructed global strong solutions in $L^{2}$-norm. Xu et al. [17] took a pure energy method to prove the fast time decay rates for the higher-order spatial derivative of solutions when the initial data are close to a stable equilibrium state in 
$H^{3}\left(\mathbb{R}^{3}\right) \cap H^{-s}\left(\mathbb{R}^{3}\right)$ for some $s \in\left[0, \frac{3}{2}\right]$. Recently, for the case of initial data are close to a stable equilibrium state in critical Besov spaces, the unique global solvability of strong solutions to the system was established by them [18]. Obviously, system (1) becomes incompressible Hall-MHD system when $\rho=$ const and there are many interesting global results, see [19]-[24] to list only a few.

When the Hall effect term is ignored, the system (1) is reverted to the wellknown MHD system. The MHD systems have been studied by many authors (see [25]-[30]). For the corresponding full compressible MHD model, we can refer to [31] [32] [33] [34] [35] and references therein. Hu and Wang [31] constructed the solution of the initial-boundary value problem and established the global weak solutions. The global smooth solutions and their decay were given by $\mathrm{Pu}$ and Guo in [33]. He et al. [35] considered boundedness and time decay of the higher-order spatial derivatives of the smooth solutions for a full compressible Hall-MHD system.

Although important, there are few results on the large-time behaviors of the Cauchy problem to the best of our knowledge. Much more complicate nonlinear terms, quantum effect term and the Hall effect term in the system (1) lead to new difficulties in decay analysis. The main novelty is to introduce (20) to cooperate with the special structure of (1). Fortunately, we can finally establish an optimal decay results for (1) under this norm, that is to say, the unknowns $(\rho, u, \theta, B)$ near the constant steady solution $(1, \overrightarrow{0}, 1, \overrightarrow{0})$ of $(1)$ are more convenient to show.

For the main results of this paper, we have the following:

Theorem 1.1 Assume that $\left(\rho_{0}-1, u_{0}, \theta_{0}-1, B_{0}\right) \in H^{4}\left(\mathbb{R}^{3}\right) \times H^{3}\left(\mathbb{R}^{3}\right) \times H^{3}\left(\mathbb{R}^{3}\right) \times H^{3}\left(\mathbb{R}^{3}\right)$, there exists a constant $\varepsilon>0$ such that if

$$
\left\|\rho_{0}-1\right\|_{H^{4}\left(\mathbb{R}^{3}\right)}+\left\|\left(u_{0}, \theta_{0}-1, B_{0}\right)\right\|_{H^{3}\left(\mathbb{R}^{3}\right)} \leq \varepsilon,
$$

then the Cauchy problem (1)-(2) admits a unique global solution $(\rho, u, \theta, B)$ satisfying

$$
\begin{aligned}
& \|(\rho-1, u, \theta-1, B)(t)\|_{H^{3}\left(\mathbb{R}^{3}\right)}^{2}+\|\hbar \nabla \rho(t)\|_{H^{3}\left(\mathbb{R}^{3}\right)}^{2} \\
& +\int_{0}^{t}\|\nabla(u, \theta, B, \hbar \rho)(\tau)\|_{H^{3}\left(\mathbb{R}^{3}\right)}^{2} \mathrm{~d} \tau \\
& \leq C\left\|\rho_{0}-1\right\|_{H^{4}\left(\mathbb{R}^{3}\right)}^{2}+\left\|\left(u_{0}, \theta_{0}-1, B_{0}\right)\right\|_{H^{3}\left(\mathbb{R}^{3}\right)}^{2} .
\end{aligned}
$$

Moreover, if $\left(\rho_{0}-1, u_{0}, \theta_{0}-1, B_{0}\right) \in L^{1}\left(\mathbb{R}^{3}\right)$, then we have

$$
\begin{gathered}
\|(\rho-1, u, \theta-1, B)(t)\|_{L^{p}\left(\mathbb{R}^{3}\right)} \leq C_{0}(1+t)^{-\frac{3}{2}\left(1-\frac{1}{p}\right)}, \quad 2 \leq p \leq 6, \\
\|\nabla(\rho-1, u, \theta-1, B)(t)\|_{H^{2}\left(\mathbb{R}^{3}\right)} \leq C_{0}(1+t)^{-\frac{5}{4}} \\
\left\|\hbar \nabla^{2}(\rho-1)\right\|_{H^{2}\left(\mathbb{R}^{3}\right)} \leq C_{0}(1+t)^{-\frac{5}{4}}
\end{gathered}
$$




$$
\begin{gathered}
\|(\rho-1, u, \theta-1, B)(t)\|_{L^{\infty}\left(\mathbb{R}^{3}\right)} \leq C_{0}(1+t)^{-\frac{5}{4}}, \\
\left\|\partial_{t}(\rho-1, u, \theta-1, B)(t)\right\|_{L^{2}\left(\mathbb{R}^{3}\right)} \leq C_{0}(1+t)^{-\frac{5}{4}},
\end{gathered}
$$

for some positive constant $C_{0}$.

Notation. Throughout this paper, the norms in the Sobolev Spaces $H^{m}\left(\mathbb{R}^{3}\right)$ and $W^{m, p}\left(\mathbb{R}^{3}\right)$ are denoted respectively by $\|\cdot\|_{H^{m}}$ and $\|\cdot\|_{W^{m, p}}$ for $m \geq 0$ and $p \geq 1$. In particular, when $m=0$, we will simply use $\|\cdot\|_{L^{2}}$ and $\|\cdot\|_{L^{p}}$. Moreover,

$$
\nabla=\left(\partial_{1}, \partial_{2}, \partial_{3}\right), \quad \partial_{i}=\partial_{x_{i}}, \quad i=1,2,3,
$$

and for any integer $\ell \geq 0, \nabla^{\ell} f$ denotes all derivatives of order $\ell$ of the function $f$. In addition, $\langle\cdot, \cdot\rangle$ denotes the inner product in $L^{2}\left(\mathbb{R}^{3}\right)$, i.e., for $f$ and $g \in L^{2}\left(\mathbb{R}^{3}\right)$,

$$
\langle f, g\rangle:=\int_{\mathbb{R}^{3}} f g \mathrm{~d} x
$$

First of all, we rewrite the Cauchy problem (1)-(2) into a more suitable form. Secondlly, we do a priori estimate and establish the global existence of solutions. Then, based on some $L^{p}-L^{q}$ estimates by the linearized operator, we prove the decay rates.

\section{Reformations}

In this subsection, we first reformulate the problem as follows. Set

$$
\tilde{\rho}=\rho-1, \quad \tilde{u}=u, \quad \tilde{\theta}=\theta-1, \quad \tilde{B}=B,
$$

then $(\tilde{\rho}, \tilde{u}, \tilde{\theta}, \tilde{B})$ takes the form

$$
\left\{\begin{array}{l}
\tilde{\rho}_{t}+\nabla \cdot \tilde{u}=\tilde{S}_{1}, \\
\tilde{u}_{t}+P_{\rho}(1,1) \nabla \tilde{\rho}+P_{\theta}(1,1) \nabla \tilde{\theta}-\frac{\hbar^{2}}{4} \nabla \Delta \tilde{\rho}-\mu \Delta \tilde{u}-(\lambda+\mu) \nabla \nabla \cdot \tilde{u}=\tilde{S}_{2}, \\
\tilde{\theta}_{t}-\frac{\kappa}{C_{v}} \Delta \tilde{\theta}+\frac{P_{\theta}(1,1)}{C_{v}} \nabla \cdot \tilde{u}=\tilde{S}_{3}, \\
\tilde{B} t-\Delta \tilde{B}=\tilde{S}_{4}, \quad \nabla \cdot \tilde{B}=0, \\
\left.(\tilde{\rho}, \tilde{u}, \tilde{\theta}, \tilde{B})\right|_{t=0}=\left(\rho_{0}-1, u_{0}, \theta_{0}-1, B_{0}\right)(x) \rightarrow(0, \overrightarrow{0}, 0, \overrightarrow{0}), \quad \text { as }|x| \rightarrow \infty,
\end{array}\right.
$$

where

$$
\begin{gathered}
\tilde{S}_{1}=-\nabla \cdot(\tilde{\rho} \tilde{u}), \\
\tilde{S}_{2}=-(\tilde{u} \cdot \nabla) \tilde{u}-\left[\frac{P_{\rho}(\tilde{\rho}+1, \tilde{\theta}+1)}{\tilde{\rho}+1}-P_{\rho}(1,1)\right] \nabla \tilde{\rho} \\
-\left[\frac{P_{\theta}(\tilde{\rho}+1, \tilde{\theta}+1)}{\tilde{\rho}+1}-P_{\theta}(1,1)\right] \nabla \tilde{\theta}-\frac{\hbar^{2}}{4}\left(\frac{|\nabla \tilde{\rho}|^{2} \nabla \tilde{\rho}}{(\tilde{\rho}+1)^{3}}-\frac{\nabla \tilde{\rho} \Delta \tilde{\rho}}{(\tilde{\rho}+1)^{2}}\right) \\
-\frac{\tilde{\rho}}{\tilde{\rho}+1}((\mu+\lambda) \nabla \nabla \cdot \tilde{u}+\mu \Delta \tilde{u})+\frac{\tilde{B} \cdot \nabla \tilde{B}}{\tilde{\rho}+1},
\end{gathered}
$$




$$
\begin{gathered}
\tilde{S}_{3}=-(\tilde{u} \cdot \nabla) \tilde{\theta}-\left[\frac{(\tilde{\theta}+1) P_{\theta}(\tilde{\rho}+1, \tilde{\theta}+1)}{C_{v}(\tilde{\rho}+1)}-\frac{P_{\theta}(1,1)}{C_{v}}\right] \nabla \cdot \tilde{u}-\frac{\tilde{\rho}}{\tilde{\rho}+1} \cdot \frac{\kappa}{C_{v}} \Delta \tilde{\theta} \\
+\frac{1}{C_{v}(\tilde{\rho}+1)}\left[2 \mu|D(\tilde{u})|^{2}+\lambda(\nabla \cdot \tilde{u})^{2}\right]+\frac{1}{C_{v}(\tilde{\rho}+1)}|\nabla \times \tilde{B}|^{2}, \\
\tilde{S}_{4}=-\tilde{u} \cdot \nabla \tilde{B}+\tilde{B} \cdot \nabla \tilde{u}-\nabla \times((\nabla \times \tilde{B}) \times \tilde{B}) .
\end{gathered}
$$

To obtain a symmetric system, we denote

$$
n=\tilde{\rho}, \quad v=\frac{1}{\sqrt{P_{\rho}(1,1)}} \tilde{u}, \quad z=\sqrt{\frac{C_{v}}{P_{\rho}(1,1)}} \tilde{\theta}, \quad B=\tilde{B},
$$

and

$$
\gamma_{1}=\sqrt{P_{\rho}(1,1)}, \quad \gamma_{2}=\frac{P_{\theta}(1,1)}{\sqrt{C_{v}}}, \quad \gamma_{3}=\frac{1}{\sqrt{C_{v}}}, \quad \kappa_{1}=\frac{\kappa}{C_{v}} .
$$

(11) can be rewritten in the perturbation form as follows

$$
\left\{\begin{array}{l}
n_{t}+\gamma_{1} \nabla \cdot v=S_{1}, \\
v_{t}+\gamma_{1} \nabla n+\gamma_{2} \nabla z-\frac{\hbar^{2}}{4 \gamma_{1}} \nabla \Delta n-\mu \Delta v-(\lambda+\mu) \nabla \nabla \cdot v=S_{2}, \\
z_{t}-\kappa_{1} \Delta z+\gamma_{2} \nabla \cdot v=S_{3}, \\
B_{t}-\Delta B=S_{4}, \quad \nabla \cdot B=0, \\
\left.(n, v, z, B)\right|_{t=0}=\left(n_{0}, v_{0}, z_{0}, B_{0}\right)(x) \rightarrow(0, \overrightarrow{0}, 0, \overrightarrow{0}), \quad \text { as }|x| \rightarrow \infty,
\end{array}\right.
$$

where the source terms $\left(S_{1}, S_{2}, S_{3}, S_{4}\right)$ are

$$
\begin{gathered}
S_{1}=-\gamma_{1} \nabla \cdot(n v), \\
S_{2}=-\gamma_{1}(v \cdot \nabla) v-h_{1}(n, z) \nabla n-h_{2}(n, z) \nabla z+\frac{\hbar^{2}}{4 \gamma_{1}}\left(\frac{|\nabla n|^{2} \nabla n}{(n+1)^{3}}-\frac{\nabla n \Delta n}{(n+1)^{2}}\right. \\
\left.-\frac{\nabla n \cdot \nabla^{2} n}{(n+1)^{2}}-g(n) \nabla \Delta n\right)-g(n)((\mu+\lambda) \nabla \nabla \cdot v+\mu \Delta v)+\frac{B \cdot \nabla B}{\gamma_{1}(n+1)}, \\
S_{3}=-\gamma_{1}(v \cdot \nabla) z-h_{3}(n, z) \nabla \cdot v-\kappa_{1} g(n) \Delta z \\
+\gamma_{3} f(n)\left[2 \mu|D(v)|^{2}+\lambda(\nabla \cdot v)^{2}\right]+\frac{\gamma_{3}}{\gamma_{1}} f(n)|\nabla \times B|^{2}, \\
S_{4}=-\gamma_{1} v \cdot \nabla B+\gamma_{1} B \cdot \nabla v-\nabla \times((\nabla \times B) \times B),
\end{gathered}
$$

and

$$
\begin{aligned}
& f(n)=\frac{1}{n+1}, \quad g(n)=\frac{n}{n+1}, \quad h_{1}(n, z)=\frac{P_{\rho}\left(n+1, \frac{\gamma_{1} z}{\sqrt{C_{v}}}+1\right)}{\gamma_{1}(n+1)}-\gamma_{1}, \\
& h_{2}(n, z)=\frac{P_{\theta}\left(n+1, \frac{\gamma_{1} z}{\sqrt{C_{v}}}+1\right)}{\sqrt{C_{v}}(n+1)}-\gamma_{2},
\end{aligned}
$$




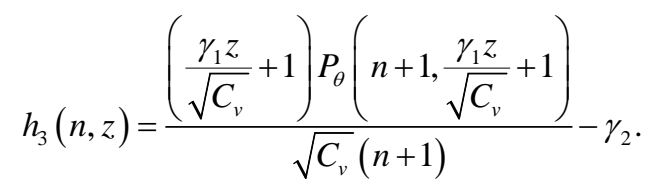

We will obtain a global solution by a combination of the local existence result and a priori estimates.

Proposition 2.1 (Local existence). Let $\left(n_{0}, v_{0}, z_{0}, B_{0}, \hbar \nabla n_{0}\right) \in H^{3}\left(\mathbb{R}^{3}\right)$ be such that

$$
\inf _{x \in \mathbb{R}^{3}}\left\{n_{0}+1, z_{0}+1\right\}>0 .
$$

There exists a positive constant $T_{0}$ depending on $N(0,0)$ and $(n, v, z, B, \hbar \nabla n) \in X\left(0, T_{0}\right)$ satisfies $N\left(0, T_{0}\right) \leq 2 N(0,0)$

$$
\begin{aligned}
X(0, T)=\{ & (n, v, z, B) \mid n, B \in C^{0}\left(0, T ; H^{3}\left(\mathbb{R}^{3}\right)\right) \cap C^{1}\left(0, T ; H^{2}\left(\mathbb{R}^{3}\right)\right), \\
& \hbar \nabla n \in C^{0}\left(0, T ; H^{3}\left(\mathbb{R}^{3}\right)\right) \cap C^{1}\left(0, T ; H^{2}\left(\mathbb{R}^{3}\right)\right), \\
& \left.v, z \in C^{0}\left(0, T ; H^{3}\left(\mathbb{R}^{3}\right)\right) \cap C^{1}\left(0, T ; H^{1}\left(\mathbb{R}^{3}\right)\right)\right\},
\end{aligned}
$$

and

$$
\begin{aligned}
N(0, T)^{2}= & \sup _{0 \leq t \leq T}\|(n, v, z, B)(t)\|_{H^{3}}^{2}+\sup _{0 \leq t \leq T}\|\hbar \nabla n(t)\|_{H^{3}}^{2} \\
& +\int_{0}^{T}\|\nabla(n, v, z, B)(\tau)\|_{H^{3}}^{2} \mathrm{~d} \tau,
\end{aligned}
$$

for any $0 \leq T \leq \infty$. Then

$$
\inf _{x \in \mathbb{R}^{3}, 0 \leq t \leq T}\{n(x, t)+1, z(x, t)+1\}>0 .
$$

Proof.

The proof can be done by using the standard iteration arguments. Refer, for instance, to [36] [37] [38].

Proposition 2.2. (A priori estimate). Let $\left(n_{0}, v_{0}, z_{0}, B_{0}, \hbar \nabla n_{0}\right) \in H^{3}\left(\mathbb{R}^{3}\right)$. $(n, v, z, B, \hbar \nabla n)$ is a solution of the initial value problem (12) on the time interval $[0, T]$. For any fixed $T>0$, then we have

$$
\sup _{0 \leq t \leq T}\|(n, v, z, B, \hbar \nabla n)(t)\|_{H^{3}} \leq \delta,
$$

the following a priori estimate holds for $t \in[0, T]$

$$
\begin{aligned}
& \|(n, v, z, B)(t)\|_{H^{3}}^{2}+\|\hbar \nabla n(t)\|_{H^{3}}^{2}+\int_{0}^{t}\|\nabla(v, z, B, \hbar n)(\tau)\|_{H^{3}}^{2} \mathrm{~d} \tau \\
& \leq \tilde{C}_{1}\left(\left\|n_{0}\right\|_{H^{4}}^{2}+\left\|\left(v_{0}, z_{0}, B_{0}\right)\right\|_{H^{3}}^{2}\right),
\end{aligned}
$$

where $\delta>0$ and $\tilde{C}_{1}$ are independent of $T$.

Remark 2.1. The global existence and uniqueness of the solution stated in Theorem 1.1 follows from Proposition 2.1 and 2.2.

Proposition 2.3. (Decay rates). Under the assumptions of Proposition 2.2, if $(n, v, z, B, \hbar \nabla n) \in L^{1}\left(\mathbb{R}^{3}\right)$, for any $t \in[0, T]$, there exists a constant $C_{1}^{\prime}$ such that 


$$
\begin{gathered}
\|(n, v, z, B)(t)\|_{L^{p}} \leq C_{1}^{\prime}(1+t)^{-\frac{3}{2}\left(1-\frac{1}{p}\right)}, \quad 2 \leq p \leq 6, \\
\|\nabla(n, v, z, B)(t)\|_{H^{2}\left(\mathbb{R}^{3}\right)} \leq C_{1}^{\prime}(1+t)^{-\frac{5}{4}}, \\
\left\|\hbar \nabla^{2} n\right\|_{H^{2}} \leq C_{1}^{\prime}(1+t)^{-\frac{5}{4}}, \\
\|(n, v, z, B)(t)\|_{L^{\infty}} \leq C_{1}^{\prime}(1+t)^{-\frac{5}{4}}, \\
\left\|\partial_{t}(n, v, z, B)(t)\right\|_{L^{2}} \leq C_{1}^{\prime}(1+t)^{-\frac{5}{4}} .
\end{gathered}
$$

\section{Energy Estimates}

This section is devoted to the proof of Proposition 2.2. We deduce energy estimates that play an important role for establishing the global existence of solutions under the problem (12).

$$
\|(n, v, z, B)(t)\|_{H^{3}}^{2}+\|\hbar \nabla n(t)\|_{H^{3}}^{2} \leq \delta .
$$

By Lemma A.2, which yields directly

$$
\frac{1}{2} \leq n+1, z+1 \leq 2, \quad\left\|(n, v, z, B), \nabla(n, v, z, B), \hbar^{2} \nabla^{2} n\right\|_{L^{\infty}} \leq C \delta .
$$

Hence, we immediately have

$$
\begin{gathered}
|f(n)| \leq C, \quad|g(n)| \leq C|n|, \quad\left|h_{i}(n, z)\right| \leq C(|n|+|z|), \\
|\nabla f(n)|,|\nabla g(n)| \leq|\nabla n|, \quad\left|\nabla^{2} f(n)\right|,\left|\nabla^{2} g(n)\right| \leq|\nabla n|^{2}+\left|\nabla^{2} n\right|, \\
\left|\nabla h_{i}(n, z)\right| \leq|\nabla n|+|\nabla z|,\left|\nabla^{2} h_{i}(n, z)\right| \leq|\nabla n|^{2}+|\nabla z|^{2}+\left|\nabla^{2} n\right|+\left|\nabla^{2} z\right|, i=1,2,3 .
\end{gathered}
$$

Before proving Proposition 2.2, we need Lemmas 3.1, 3.2 and 3.3.

Lemma 3.1 Let $(n, v, z, B)$ be defined in (12), then it holds that

$$
\begin{aligned}
& \frac{1}{2} \frac{\mathrm{d}}{\mathrm{d} t}\left(\|(n, v, z, B)\|_{L^{2}}^{2}+\frac{\hbar^{2}}{4 \gamma_{1}^{2}}\|\nabla n\|_{L^{2}}^{2}\right)+C\|\nabla v\|_{L^{2}}^{2}+C\|\nabla z\|_{L^{2}}^{2}+C\|\nabla B\|_{L^{2}}^{2} \\
& \leq C \delta\left(\|\nabla n\|_{L^{2}}^{2}+\hbar^{2}\|\nabla n\|_{L^{2}}^{2}\right) .
\end{aligned}
$$

Proof. Multiplying $(12)_{1},(12)_{2},(12)_{3}$ and $(12)_{4}$ by $n, V, z$ and $B$ respectively, the integration over $\mathbb{R}^{3}$ gives

$$
\begin{aligned}
& \frac{1}{2} \frac{\mathrm{d}}{\mathrm{d} t}\|(n, v, z, B)\|_{L^{2}}^{2}+\mu\|\nabla v\|_{L^{2}}^{2}+(\mu+\lambda)\|\nabla \cdot v\|_{L^{2}}^{2}+\kappa_{1}\|\nabla z\|_{L^{2}}^{2}+\|\nabla B\|_{L^{2}}^{2} \\
& =\left\langle\frac{\hbar^{2}}{4 \gamma_{1}} \nabla \Delta n, v\right\rangle+\left\langle S_{1}, n\right\rangle+\left\langle S_{2}, v\right\rangle+\left\langle S_{3}, z\right\rangle+\left\langle S_{4}, B\right\rangle .
\end{aligned}
$$

The five terms on the right-hand side of the above equation can be estimated as follows.

Firstly, we get

$$
\left\langle\frac{\hbar^{2}}{4 \gamma_{1}} \nabla \Delta n, v\right\rangle=\left\langle\frac{\hbar^{2}}{4 \gamma_{1}} \nabla n, \Delta v\right\rangle=\left\langle\frac{\hbar^{2}}{4 \gamma_{1}} \nabla n, \frac{1}{\gamma_{1}} \nabla F_{1}-\frac{1}{\gamma_{1}} \nabla n_{t}\right\rangle
$$




$$
\begin{aligned}
& =-\left\langle\frac{\hbar^{2}}{4 \gamma_{1}^{2}} \nabla n, \nabla n_{t}\right\rangle+\left\langle\frac{\hbar^{2}}{4 \gamma_{1}} \Delta n,(\nabla n \cdot v+n \nabla \cdot v)\right\rangle \\
& \leq-\frac{1}{2} \frac{\mathrm{d}}{\mathrm{d} t}\left(\frac{\hbar^{2}}{4 \gamma_{1}^{2}}\|\nabla n\|_{L^{2}}^{2}\right)+C \hbar^{2}\|\Delta n\|_{L^{3}}\left(\|\nabla n\|_{L^{2}}\|v\|_{L^{6}}+\|\nabla v\|_{L^{2}}\|n\|_{L^{6}}\right) \\
& \leq-\frac{1}{2} \frac{\mathrm{d}}{\mathrm{d} t}\left(\frac{\hbar^{2}}{4 \gamma_{1}^{2}}\|\nabla n\|_{L^{2}}^{2}\right)+C \delta \hbar^{2}\left(\|\nabla n\|_{L^{2}}^{2}+\|\nabla v\|_{L^{2}}^{2}\right) .
\end{aligned}
$$

Secondly, we obtain

$$
\begin{aligned}
\left\langle S_{1}, n\right\rangle & =-\left\langle\gamma_{1} n \nabla \cdot v, n\right\rangle-\left\langle\gamma_{1} v \cdot \nabla n, n\right\rangle \\
& \leq \gamma_{1}\|n\|_{L^{3}}\|\nabla \cdot v\|_{L^{2}}\|n\|_{L^{6}}+\gamma_{1}\|v\|_{L^{6}}\|\nabla n\|_{L^{2}}\|n\|_{L^{3}} \\
& \leq C \delta\left(\|\nabla n\|_{L^{2}}^{2}+\|\nabla v\|_{L^{2}}^{2}\right) .
\end{aligned}
$$

Next, we have

$$
\begin{aligned}
\left\langle S_{2}, v\right\rangle= & -\left\langle\gamma_{1} v \cdot \nabla v, v\right\rangle-\left\langle h_{1}(n, z) \nabla n, v\right\rangle-\left\langle h_{2}(n, z) \nabla z, v\right\rangle \\
& +\left\langle\frac{\hbar^{2}}{4 \gamma_{1}}\left(\frac{|\nabla n|^{2} \nabla n}{(n+1)^{3}}-\frac{\nabla n \Delta n}{(n+1)^{2}}-\frac{\nabla n \cdot \nabla^{2} n}{(n+1)^{2}}-g(n) \nabla \Delta n\right), v\right\rangle \\
& -\langle g(n)((\mu+\lambda) \nabla \nabla \cdot v+\mu \Delta v), v\rangle+\left\langle\frac{B \cdot \nabla B}{\gamma_{1}(n+1)}, v\right\rangle \\
:= & G_{1}+G_{2}+G_{3}+G_{4}+G_{5}+G_{6} .
\end{aligned}
$$

it follows from (20) and (21) that

$$
\begin{aligned}
G_{1}+G_{2}+G_{3} \leq & C\|v\|_{L^{3}}\|\nabla v\|_{L^{2}}\|v\|_{L^{6}}+C\left\|h_{1}(n, z)\right\|_{L^{3}}\|\nabla n\|_{L^{2}}\|v\|_{L^{6}} \\
& +C\left\|h_{2}(n, z)\right\|_{L^{3}}\|\nabla z\|_{L^{2}}\|v\|_{L^{6}} \\
\leq & C \delta\left(\|\nabla n\|_{L^{2}}^{2}+\|\nabla v\|_{L^{2}}^{2}+\|\nabla z\|_{L^{2}}^{2}\right) .
\end{aligned}
$$

To deal with the term $G_{4}$, we arrive at

$$
\begin{aligned}
G_{4}= & \left\langle\frac{\hbar^{2}}{4 \gamma_{1}}\left(\frac{|\nabla n|^{2} \nabla n}{(n+1)^{3}}-\frac{\nabla n \Delta n}{(n+1)^{2}}-\frac{\nabla n \cdot \nabla^{2} n}{(n+1)^{2}}\right), v\right\rangle \\
& +\frac{\hbar^{2}}{4 \gamma_{1}}\left\langle g^{\prime}(n) \cdot v, \Delta n\right\rangle+\frac{\hbar^{2}}{4 \gamma_{1}}\langle g(n) \nabla \cdot v, \Delta n\rangle \\
\leq & C \hbar^{2}\left(\left\|\frac{\nabla n}{(n+1)^{3}}\right\|_{L^{\infty}}\|\nabla n\|_{L^{2}}\|\nabla n\|_{L^{3}}\|v\|_{L^{6}}+\left\|\frac{1}{(n+1)^{2}}\right\|_{L^{\infty}}\|\nabla n\|_{L^{2}}\|\Delta n\|_{L^{3}}\|v\|_{L^{6}}\right. \\
& +\left\|\frac{1}{(n+1)^{2}}\right\|_{L^{\infty}}\|\nabla n\|_{L^{2}}\left\|\nabla^{2} n\right\|_{L^{3}}\|v\|_{L^{6}}+\left\|g^{\prime}(n)\right\|_{L^{2}}\|v\|_{L^{6}}\|\Delta n\|_{L^{3}} \\
& \left.+\|g(n)\|_{L^{3}}\|\Delta n\|_{L^{6}}\|\nabla \cdot v\|_{L^{2}}\right) \\
\leq & C \delta \hbar^{2}\left(\|\nabla n\|_{L^{2}}^{2}+\|\nabla v\|_{L^{2}}^{2}\right) .
\end{aligned}
$$

Let $G_{5}=G_{51}+G_{52}$. For $G_{52}$, we have by (20), (21), the Hölder inequality, Young inequality, Lemma A.1 and integration by parts that 


$$
\begin{aligned}
G_{52} & =\mu\langle\nabla(g(n) v), \nabla v\rangle \\
& \leq C\left\langle g^{\prime}(n) \nabla n \cdot v, \nabla v\right\rangle+C\langle g(n) \nabla \cdot v, \nabla v\rangle \\
& \leq C\|\nabla v\|_{L^{2}}^{2}\|g(n)\|_{L^{\infty}}+C\|\nabla v\|_{L^{2}}\|\nabla n\|_{L^{2}}\left\|g^{\prime}(n)\right\|_{L^{\infty}}\|v\|_{L^{\infty}} \\
& \leq C \delta\left(\|\nabla n\|_{L^{2}}^{2}+\|\nabla v\|_{L^{2}}^{2}\right) .
\end{aligned}
$$

Similarly to the proof of $G_{52}$, we have

$$
G_{51} \leq C \delta\left(\|\nabla n\|_{L^{2}}^{2}+\|\nabla v\|_{L^{2}}^{2}\right) .
$$

We similarly obtain

$$
G_{6} \leq C \delta\left(\|\nabla v\|_{L^{2}}^{2}+\|\nabla B\|_{L^{2}}^{2}\right) .
$$

In light of the estimates $G_{1} \sim G_{6}$, we see that

$$
\left\langle S_{2}, v\right\rangle \leq C \delta \hbar^{2}\left(\|\nabla n\|_{L^{2}}^{2}+\hbar^{2}\|\nabla v\|_{L^{2}}^{2}+\|\nabla z\|_{L^{2}}^{2}+\|\nabla B\|_{L^{2}}^{2}\right) .
$$

For the fourth term, we have

$$
\begin{aligned}
\left\langle S_{3}, z\right\rangle= & -\left\langle\gamma_{1} v \cdot \nabla z, z\right\rangle-\left\langle h_{3}(n, z) \nabla \cdot v, z\right\rangle-\left\langle\kappa_{1} g(n) \Delta z, z\right\rangle \\
& +\gamma_{3}\left\langle f(n)\left[2 \mu|D(v)|^{2}+\lambda(\nabla \cdot v)^{2}\right], z\right\rangle+\frac{\gamma_{3}}{\gamma_{1}}\left\langle f(n)|\nabla \times B|^{2}, z\right\rangle \\
& :=L_{1}+L_{2}+L_{3}+L_{4}+L_{5} .
\end{aligned}
$$

It follows from Hölder's inequality, Lemma A.1 and (20) that

$$
L_{1} \leq \gamma_{1}\|v\|_{L^{3}}\|\nabla z\|_{L^{2}}\|z\|_{L^{6}} \leq C \delta\|\nabla z\|_{L^{2}}^{2} .
$$

In the same way as above, we know

$$
L_{2} \leq C\left\|h_{3}(n, z)\right\|_{L^{3}}\|\nabla \cdot v\|_{L^{2}}\|z\|_{L^{6}} \leq C \delta\left(\|\nabla v\|_{L^{2}}^{2}+\|\nabla z\|_{L^{2}}^{2}\right) .
$$

Similarly,

$$
L_{3} \leq C\left\|g^{\prime}(n)\right\|_{L^{2}}\|\nabla z\|_{L^{2}}\|z\|_{L^{6}}+C\|\nabla z\|_{L^{2}}^{2}\|g(n)\|_{L^{\infty}} \leq C \delta\|\nabla z\|_{L^{2}}^{2} .
$$

Let $L_{4}=L_{41}+L_{42}$, similarly

$$
L_{41}=2 \mu \gamma_{3}\left\langle f(n)|D(v)|^{2}, z\right\rangle \leq C\|f(n)\|_{L^{3}}\|\nabla v\|_{L^{2}}^{2}\|z\|_{L^{6}} \leq C \delta\left(\|\nabla v\|_{L^{2}}^{2}+\|\nabla z\|_{L^{2}}^{2}\right) .
$$

In a similar way,

$$
L_{42} \leq C \delta\left(\|\nabla v\|_{L^{2}}^{2}+\|\nabla z\|_{L^{2}}^{2}\right)
$$

We have

$$
L_{4} \leq C \delta\left(\|\nabla v\|_{L^{2}}^{2}+\|\nabla z\|_{L^{2}}^{2}\right) .
$$

A similar argument shows that

$$
\begin{aligned}
L_{5} & =\frac{\gamma_{3}}{\gamma_{1}}\left\langle f(n)|\nabla \times B|^{2}, z\right\rangle \\
& \leq C\|f(n)\|_{L^{3}}\|\nabla B\|_{L^{2}}^{2}\|z\|_{L^{6}} \\
& \leq C \delta\left(\|\nabla z\|_{L^{2}}^{2}+\|\nabla B\|_{L^{2}}^{2}\right) .
\end{aligned}
$$


Summing up $L_{1} \sim L_{5}$, we can get

$$
\left\langle S_{3}, z\right\rangle \leq C \delta\left(\|\nabla v\|_{L^{2}}^{2}+\|\nabla z\|_{L^{2}}^{2}+\|\nabla B\|_{L^{2}}^{2}\right) .
$$

Finally, we have

$$
\left\langle S_{4}, B\right\rangle=-\left\langle\gamma_{1} v \cdot \nabla B, B\right\rangle+\left\langle\gamma_{1} B \cdot \nabla v, B\right\rangle-\langle\nabla \times((\nabla \times B) \times B), B\rangle .
$$

In a similar way, we know

$$
-\left\langle\gamma_{1} v \cdot \nabla B, B\right\rangle+\left\langle\gamma_{1} B \cdot \nabla v, B\right\rangle \leq C \delta\left(\|\nabla v\|_{L^{2}}^{2}+\|\nabla B\|_{L^{2}}^{2}\right) .
$$

By a direct computation, we have

$$
-\langle\nabla \times((\nabla \times B) \times B), B\rangle=\langle(\nabla \times B) \times B, \nabla \times B\rangle=0 .
$$

We get

$$
\left\langle S_{4}, B\right\rangle \leq C \delta\left(\|\nabla v\|_{L^{2}}^{2}+\|\nabla B\|_{L^{2}}^{2}\right) .
$$

Plugging these estimates into (23), we deduce (22).

Then, we give a energy estimate of the higher-order for $(n, v, z, B)$.

Lemma 3.2. Let $(n, v, z, B)$ be defined in (12), then we have

$$
\begin{aligned}
& \frac{\mathrm{d}}{\mathrm{d} t}\left(\|\nabla(n, v, z, B)\|_{H^{2}}^{2}+\hbar^{2}\left\|\nabla^{2} n\right\|_{H^{2}}^{2}\right)+C\left\|\nabla^{2} v\right\|_{H^{2}}^{2}+C\left\|\nabla^{2} z\right\|_{H^{2}}^{2}+C\left\|\nabla^{2} B\right\|_{H^{2}}^{2} \\
& \leq C \delta\left(\|\nabla n\|_{H^{2}}^{2}+\hbar^{2}\left\|\nabla^{2} n\right\|_{H^{2}}^{2}+\|\nabla v\|_{L^{2}}^{2}+\|\nabla z\|_{L^{2}}^{2}+\|\nabla B\|_{L^{2}}^{2}\right) .
\end{aligned}
$$

Proof. Applying $\nabla^{k+1}$ with $0 \leq k \leq 2$ to (12) and then taking $L^{2}$-inner product with $\left(\nabla^{k+1} n, \nabla^{k+1} v, \nabla^{k+1} z, \nabla^{k+1} B\right)$, we obtain

$$
\begin{aligned}
& \frac{1}{2} \frac{\mathrm{d}}{\mathrm{d} t}\left\|\nabla^{k+1}(n, v, z, B)\right\|_{L^{2}}^{2}+\mu\left\|\nabla^{k+2} v\right\|_{L^{2}}^{2}+(\lambda+\mu)\left\|\nabla^{k+1} \nabla \cdot v\right\|_{L^{2}}^{2} \\
+ & \kappa_{1}\left\|\nabla^{k+2} z\right\|_{L^{2}}^{2}+\left\|\nabla^{k+2} B\right\|_{L^{2}}^{2} \\
= & \left\langle\frac{\hbar^{2}}{4 \gamma_{1}} \nabla^{k+1}\left(\frac{1}{n+1} \nabla \Delta n\right), \nabla^{k+1} v\right\rangle-\left\langle\gamma_{1} \nabla^{k+1} \nabla \cdot(n v), \nabla^{k+1} n\right\rangle \\
& -\left\langle\nabla^{k+1}\left(\gamma_{1} v \cdot \nabla v\right), \nabla^{k+1} v\right\rangle-\left\langle\nabla^{k+1}\left(h_{1}(n, z) \nabla n\right), \nabla^{k+1} v\right\rangle \\
& -\left\langle\nabla^{k+1}\left(h_{2}(n, z) \nabla z\right), \nabla^{k+1} v\right\rangle \\
& +\left\langle\frac{\hbar^{2}}{4 \gamma_{1}} \nabla^{k+1}\left(-\frac{\nabla n \cdot \nabla^{2} n}{(n+1)^{2}}-\frac{\nabla n \Delta n}{(n+1)^{2}}+\frac{|\nabla n|^{2} \nabla n}{(n+1)^{3}}\right), \nabla^{k+1} v\right\rangle \\
& -\left\langle\nabla^{k+1}(g(n)((\mu+\lambda) \nabla \nabla \cdot v)+\mu \Delta v), \nabla^{k+1} v\right\rangle \\
& +\left\langle\nabla^{k+1}\left(\frac{B \cdot \nabla B}{\gamma_{1}(n+1)}\right), \nabla^{k+1} v\right\rangle-\left\langle\nabla^{k+1}\left(\gamma_{1} v \cdot \nabla z\right), \nabla^{k+1} z\right\rangle \\
& -\left\langle\nabla^{k+1}\left(h_{3}(n, z) \nabla \cdot v\right), \nabla^{k+1} z\right\rangle-\kappa_{1}\left\langle\nabla^{k+1}(g(n) \Delta z), \nabla^{k+1} z\right\rangle \\
& +\gamma_{3}\left\langle\nabla^{k+1}\left\{f(n)\left[2 \mu|D(v)|^{2}+\lambda(\nabla \cdot v)^{2}\right]\right\}, \nabla^{k+1} z\right\rangle \\
& +\frac{\gamma_{3}}{\gamma_{1}}\left\langle\nabla^{k+1}\left[f(n)|\nabla \times B|^{2}\right], \nabla^{k+1} z\right\rangle
\end{aligned}
$$




$$
\begin{aligned}
& -\left\langle\nabla^{k+1}\left(\gamma_{1} v \cdot \nabla B\right), \nabla^{k+1} B\right\rangle+\left\langle\nabla^{k+1}\left(\gamma_{1} B \cdot \nabla v\right), \nabla^{k+1} B\right\rangle \\
& -\left\langle\nabla^{k+1}(\nabla \times((\nabla \times B) \times B)), \nabla^{k+1} B\right\rangle \\
: & H_{1}+H_{2}+H_{3}+H_{4}+H_{5}+H_{6}+H_{7}+H_{8}+H_{9} \\
& +H_{10}+H_{11}+H_{12}+H_{13}+H_{14}+H_{15}+H_{16} .
\end{aligned}
$$

First of all, $H_{1}$ is written as

$$
\begin{aligned}
H_{1} & =\frac{\hbar^{2}}{4 \gamma_{1}}\left\langle\nabla^{k+1} v, \frac{1}{n+1} \nabla^{k+1} \nabla \Delta n\right\rangle+\frac{\hbar^{2}}{4 \gamma_{1}} \sum_{1 \leq l \leq k+1} C_{k+1}^{l}\left\langle\nabla^{k+1} v, \nabla^{l}\left(\frac{1}{n+1}\right) \nabla^{k+1-l} \nabla \Delta n\right\rangle \\
& :=H_{1}^{1}+H_{1}^{2} .
\end{aligned}
$$

The first term $H_{1}^{1}$ can be rewritten as

$$
\begin{aligned}
H_{1}^{1}= & -\frac{\hbar^{2}}{4 \gamma_{1}}\left\langle\nabla^{k+1} v, \nabla\left(\frac{1}{n+1}\right) \nabla^{k+1} \Delta n\right\rangle-\frac{\hbar^{2}}{4 \gamma_{1}}\left\langle\nabla^{k+1} \nabla \cdot v, \frac{1}{n+1} \nabla^{k+1} \Delta n\right\rangle \\
= & \frac{\hbar^{2}}{4 \gamma_{1}}\left\langle\nabla^{2}\left(\frac{1}{n+1}\right) \nabla^{k+2} n, \nabla^{k+1} v\right\rangle+\frac{\hbar^{2}}{4 \gamma_{1}}\left\langle\nabla\left(\frac{1}{n+1}\right) \nabla^{k+2} n, \nabla^{k+2} v\right\rangle \\
& -\frac{\hbar^{2}}{4 \gamma_{1}}\left\langle\frac{1}{n+1} \nabla^{k+1} \Delta n, \nabla^{k+1} \nabla \cdot v\right\rangle,
\end{aligned}
$$

where the first and two terms can be estimated as

$$
\begin{aligned}
& \frac{\hbar^{2}}{4 \gamma_{1}}\left\langle\nabla^{2}\left(\frac{1}{n+1}\right) \nabla^{k+2} n, \nabla^{k+1} v\right\rangle+\frac{\hbar^{2}}{4 \gamma_{1}}\left\langle\nabla\left(\frac{1}{n+1}\right) \nabla^{k+2} n, \nabla^{k+2} v\right\rangle \\
& \leq C \delta \hbar^{2}\left(\left\|\nabla^{k+2} n\right\|_{L^{2}}^{2}+\left\|\nabla^{k+2} v\right\|_{L^{2}}^{2}\right) .
\end{aligned}
$$

The third term in $H_{1}^{1}$ is written as

$$
\begin{aligned}
& \frac{\hbar^{2}}{4 \gamma_{1}}\left\langle\nabla\left(\frac{1}{n+1}\right) \nabla^{k+2} n, \nabla^{k+1} \nabla \cdot v\right\rangle+\frac{\hbar^{2}}{4 \gamma_{1}}\left\langle\frac{1}{n+1} \nabla^{k+2} n, \nabla^{k+2} \nabla \cdot v\right\rangle \\
= & \frac{\hbar^{2}}{4 \gamma_{1}}\left\langle\nabla\left(\frac{1}{n+1}\right) \nabla^{k+2} n, \nabla^{k+1} \nabla \cdot v\right\rangle-\frac{\hbar^{2}}{4 \gamma_{1}^{2}}\left\langle\frac{1}{(n+1)^{2}} \nabla^{k+2} n, \nabla^{k+2} n_{t}\right\rangle \\
& -\frac{\hbar^{2}}{4 \gamma_{1}^{2}} \sum_{0 \leq l \leq k+1} C_{k+2}^{l}\left\langle\frac{1}{n+1} \nabla^{l} n_{t} \nabla^{k+2-l}\left(\frac{1}{n+1}\right), \nabla^{k+2} n\right\rangle \\
& -\frac{\hbar^{2}}{4 \gamma_{1}}\left\langle\frac{1}{n+1} \nabla^{k+2} n, \nabla^{k+2}\left(\frac{\nabla n \cdot v}{n+1}\right)\right\rangle \\
: & =I_{1}+I_{2}+I_{3}+I_{4} .
\end{aligned}
$$

Moreover, $I_{1}$ and $I_{2}$ can be estimated similarly

$$
I_{1}+I_{2} \leq-\frac{1}{2} \cdot \frac{\hbar^{2}}{4 \gamma_{1}^{2}} \frac{\mathrm{d}}{\mathrm{d} t}\left\|\frac{1}{n+1} \nabla^{k+2} n\right\|_{L^{2}}^{2}+C \delta \hbar^{2}\left(\left\|\nabla^{k+2} n\right\|_{L^{2}}^{2}+\left\|\nabla^{k+2} v\right\|_{L^{2}}^{2}\right) .
$$

For the term $I_{3}$, we see that

$$
\begin{aligned}
I_{3} \leq & C \hbar^{2}\left\|\nabla^{k+2} n\right\|_{L^{2}} \sum_{0 \leq l \leq k+1}\left\|\nabla^{l} n_{t} \nabla^{k+2-l}\left(\frac{1}{n+1}\right)\right\|_{L^{2}} \\
\leq & C \hbar^{2}\left\|\nabla^{k+2} n\right\|_{L^{2}} \sum_{0 \leq l \leq k+1}\left(\left\|\nabla^{l} \nabla \cdot v \nabla^{k+2-l}\left(\frac{1}{n+1}\right)\right\|_{L^{2}}\right. \\
& \left.+\left\|\nabla^{l}(\nabla n \cdot v) \nabla^{k+2-l}\left(\frac{1}{n+1}\right)\right\|_{L^{2}}+\left\|\nabla^{l}(n \nabla \cdot v) \nabla^{k+2-l}\left(\frac{1}{n+1}\right)\right\|_{L^{2}}\right)
\end{aligned}
$$


For the second term of $I_{3}$,

$$
\begin{aligned}
& C \hbar^{2}\left\|\nabla^{k+2} n\right\|_{L^{2}}\left(\left\|\nabla n \cdot v \nabla^{k+2}\left(\frac{1}{n+1}\right)\right\|_{L^{2}}+\left\|\nabla(\nabla n \cdot v) \nabla^{k+1}\left(\frac{1}{n+1}\right)\right\|_{L^{2}}\right. \\
& \left.+\left\|\nabla^{k}(\nabla n \cdot v) \nabla^{2}\left(\frac{1}{n+1}\right)\right\|_{L^{2}}+\left\|\nabla^{k+1}(\nabla n \cdot v) \nabla\left(\frac{1}{n+1}\right)\right\|_{L^{2}}\right) \\
& \leq C \hbar^{2}\left\|\nabla^{k+2} n\right\|_{L^{2}}\left(C \delta\left\|\nabla^{k+2} n\right\|_{L^{2}}+C \delta\left\|\nabla^{k+2} n\right\|_{L^{2}}\right. \\
& \left.+C \delta\left\|\nabla^{k}(\nabla n \cdot v)\right\|_{L^{2}}+C \delta\left\|\nabla^{k+1}(\nabla n \cdot v)\right\|_{L^{2}}\right) \\
& \leq C \delta \hbar^{2}\left\|\nabla^{k+2} n\right\|_{L^{2}}\left(\left\|\nabla^{k+2} n\right\|_{L^{2}}+\left\|\nabla^{k}(\nabla n \cdot v)\right\|_{L^{2}}+\left\|\nabla^{k+1}(\nabla n \cdot v)\right\|_{L^{2}}\right) \\
& \leq C \delta \hbar^{2}\left(\left\|\nabla^{2} n\right\|_{H^{k}}^{2}+\left\|\nabla^{k+2} v\right\|_{L^{2}}^{2}\right),
\end{aligned}
$$

where

$$
\begin{array}{rl} 
& \left\|\nabla^{k+1}(\nabla n \cdot v)\right\|_{L^{2}} \\
\leq & C \sum_{0 \leq I \leq k+1}\left\|\nabla^{l+1} n \nabla^{k+1-l} v\right\|_{L^{2}} \\
= & C\left\|\nabla n \nabla^{k+1} v\right\|_{L^{2}}+C\left\|\nabla^{2} n \nabla^{k} v\right\|_{L^{2}} \\
& +C\left\|\nabla^{k+1} n \nabla v\right\|_{L^{2}}+C\left\|\nabla^{k+2} n v\right\|_{L^{2}} \\
\leq C & C\|\nabla n\|_{L^{3}}\left\|\nabla^{k+1} v\right\|_{L^{6}}+\left\|\nabla^{2} n\right\|_{L^{2}}\left\|\nabla^{k} v\right\|_{L^{\infty}} \\
& \left.+\left\|\nabla^{k+1} n\right\|_{L^{6}}\|\nabla v\|_{L^{3}}+\left\|\nabla^{k+2} n\right\|_{L^{2}}\|v\|_{L^{\infty}}\right) \\
\leq C & C\left(\left\|\nabla^{2} n\right\|_{H^{k}}+\left\|\nabla^{k+2} v\right\|_{L^{2}}\right),
\end{array}
$$

in a similar way

$$
\left\|\nabla^{k}(\nabla n \cdot v)\right\|_{L^{2}} \leq C \delta\left(\left\|\nabla^{2} n\right\|_{H^{k}}+\left\|\nabla^{k+2} v\right\|_{L^{2}}\right) .
$$

For $I_{3}$,

$$
I_{3} \leq C \delta \hbar^{2}\left(\left\|\nabla^{2} n\right\|_{H^{k}}^{2}+\left\|\nabla^{k+2} v\right\|_{L^{2}}^{2}\right) .
$$

For the term $I_{4}$, we know

$$
\begin{aligned}
I_{4}= & -\frac{\hbar^{2}}{4 \gamma_{1}}\left\langle\nabla^{k+2} n \nabla^{k+3} n, \frac{v}{(n+1)^{2}}\right\rangle \\
& -\frac{\hbar^{2}}{4 \gamma_{1}} \sum_{0 \leq l \leq k+1} C_{k+2}^{l}\left\langle\frac{1}{n+1} \nabla^{k+2} n, \nabla^{l+1} n \nabla^{k+2-l}\left(\frac{v}{n+1}\right)\right\rangle .
\end{aligned}
$$

For the first term of $I_{4}$, after integrating by parts, we infer from (20) that

$$
\begin{aligned}
& -\frac{1}{2} \cdot \frac{\hbar^{2}}{4 \gamma_{1}}\left\langle\nabla\left(\left|\nabla^{k+2} n\right|^{2}\right), \frac{v}{(n+1)^{2}}\right\rangle \\
& =\frac{1}{2} \cdot \frac{\hbar^{2}}{4 \gamma_{1}}\left\langle\left|\nabla^{k+2} n\right|^{2}, \nabla \cdot\left(\frac{v}{(n+1)^{2}}\right)\right\rangle \leq C \delta \hbar^{2}\left\|\nabla^{k+2} n\right\|_{L^{2}}^{2} .
\end{aligned}
$$

In addition, 


$$
\begin{aligned}
& C \hbar^{2}\left\|\nabla^{k+2} n\right\|_{L^{2}} \sum_{0 \leq I \leq k+1}\left\|\nabla^{l+1} n \nabla^{k+2-l}\left(\frac{v}{n+1}\right)\right\|_{L^{2}} \\
& =C \hbar^{2}\left\|\nabla^{k+2} n\right\|_{L^{2}}\left(\left\|\nabla n \nabla^{k+2}\left(\frac{v}{n+1}\right)\right\|_{L^{2}}+\left\|\nabla^{2} n \nabla^{k+1}\left(\frac{v}{n+1}\right)\right\|_{L^{2}}\right. \\
& \left.+\left\|\nabla^{k+1} n \nabla^{2}\left(\frac{v}{n+1}\right)\right\|\left\|_{L^{2}}+\right\| \nabla^{k+2} n \nabla\left(\frac{v}{n+1}\right) \|_{L^{2}}\right) \\
& \leq C \hbar^{2}\left\|\nabla^{k+2} n\right\|_{L^{2}}\left(C \delta\left\|\nabla^{k+2}\left(\frac{v}{n+1}\right)\right\|_{L^{2}}+C \delta\left\|\nabla^{k+2} n\right\|_{L^{2}}\right) \\
& \leq C \delta \hbar^{2}\left(\left\|\nabla^{2} n\right\|_{H^{k}}^{2}+\left\|\nabla^{k+2} v\right\|_{L^{2}}^{2}\right),
\end{aligned}
$$

where

$$
\begin{aligned}
& \left\|\nabla^{k+2}\left(\frac{v}{n+1}\right)\right\|_{L^{2}} \leq C \sum_{0 \leq I \leq k+2}\left\|\nabla^{l} v \nabla^{k+2-l}\left(\frac{1}{n+1}\right)\right\|_{L^{2}} \\
& =C\left\|v \nabla^{k+2}\left(\frac{1}{n+1}\right)\right\|_{L^{2}}+C\left\|\nabla^{k+2} v\left(\frac{1}{n+1}\right)\right\|_{L^{2}} \\
& +C\left\|\nabla^{k+1} v \nabla\left(\frac{1}{n+1}\right)\right\|_{L^{2}}+C \sum_{1 \leq l \leq k}\left\|\nabla^{l} v \nabla^{k+2-l}\left(\frac{1}{n+1}\right)\right\|_{L^{2}} \\
& \leq C \delta\left(\left\|\nabla^{2} n\right\|_{H^{k}}+\left\|\nabla^{k+2} v\right\|_{L^{2}}\right) .
\end{aligned}
$$

Collecting these terms, we get

$$
H_{1}^{1} \leq-\frac{1}{2} \cdot \frac{\hbar^{2}}{4 \gamma_{1}^{2}} \frac{\mathrm{d}}{\mathrm{d} t}\left\|\frac{1}{n+1} \nabla^{k+2} n\right\|_{L^{2}}^{2}+C \delta \hbar^{2}\left(\left\|\nabla^{2} n\right\|_{H^{k}}^{2}+\left\|\nabla^{k+2} v\right\|_{L^{2}}^{2}\right) .
$$

For the second term of (35), In view of (20), (21), Hölder's inequality and Lemma A.1, there holds

$$
\begin{aligned}
H_{1}^{2}= & -\frac{\hbar^{2}}{4 \gamma_{1}} C_{k+1}^{1}\left\langle\nabla^{2}\left(\frac{1}{n+1}\right) \nabla^{k+2} n, \nabla^{k+1} v\right\rangle-\frac{\hbar^{2}}{4 \gamma_{1}} C_{k+1}^{1}\left\langle\nabla^{k+1} \nabla \cdot v, \nabla\left(\frac{1}{n+1}\right) \nabla^{k+2} n\right\rangle \\
& +\frac{\hbar^{2}}{4 \gamma_{1}} C_{k+1}^{2}\left\langle\nabla^{2}\left(\frac{1}{n+1}\right) \nabla^{k} \Delta n, \nabla^{k+1} v\right\rangle+\frac{\hbar^{2}}{4 \gamma_{1}}\left\langle\nabla^{k+1}\left(\frac{1}{n+1}\right) \nabla \Delta n, \nabla^{k+1} v\right\rangle \\
\leq & C \hbar^{2}\left(\left\|\nabla^{2}\left(\frac{1}{n+1}\right)\right\|\left\|\nabla_{L^{3}}^{k+1} v\right\|_{L^{6}}+\left\|\nabla\left(\frac{1}{n+1}\right)\right\|\left\|_{L^{\infty}}\right\| \nabla^{k+2} v \|_{L^{2}}\right)\left\|\nabla^{k+2} n\right\|_{L^{2}} \\
& +C \hbar^{2}\left(\left\|\nabla^{2}\left(\frac{1}{n+1}\right)\right\|\left\|_{L^{3}}\right\| \nabla^{k+2} n\left\|_{L^{2}}+\right\| \nabla^{k+1}\left(\frac{1}{n+1}\right)\left\|_{L^{3}}\right\| \nabla^{3} n \|_{L^{2}}\right)\left\|\nabla^{k+1} v\right\|_{L^{6}} \\
\leq & C \delta \hbar^{2}\left(\left\|\nabla^{2} n\right\|_{H^{k}}^{2}+\left\|\nabla^{k+2} n\right\|_{L^{2}}^{2}\right) .
\end{aligned}
$$

Then

$$
H_{1} \leq-\frac{1}{2} \cdot \frac{\hbar^{2}}{4 \gamma_{1}^{2}} \frac{\mathrm{d}}{\mathrm{d} t}\left\|\frac{1}{n+1} \nabla^{k+2} n\right\|_{L^{2}}^{2}+C \delta \hbar^{2}\left(\left\|\nabla^{2} n\right\|_{H^{k}}^{2}+\left\|\nabla^{k+2} v\right\|_{L^{2}}^{2}\right) .
$$

$\mathrm{H}_{2}$ can be rewritten as

$$
\begin{aligned}
H_{2} & =-\gamma_{1}\left\langle\nabla^{k+1}(\nabla n \cdot v), \nabla^{k+1} n\right\rangle-\gamma_{1}\left\langle\nabla^{k+1}(n \nabla \cdot v), \nabla^{k+1} n\right\rangle \\
& :=H_{2}^{1}+H_{2}^{2} .
\end{aligned}
$$


The first term $H_{2}^{1}$ can be bounded by

$$
\begin{aligned}
H_{2}^{1}= & -\gamma_{1}\left\langle\nabla^{k+2} n \cdot v, \nabla^{k+1} n\right\rangle-\gamma_{1} \sum_{0 \leq I \leq k}\left\langle C_{k+1}^{l} \nabla^{l+1} n \nabla^{k+1-l} v, \nabla^{k+1} n\right\rangle \\
\leq & \frac{1}{2} \gamma_{1}\left\langle\left|\nabla^{k+1} n\right|^{2}, \nabla \cdot v\right\rangle+C\left\|\nabla^{k+1} n\right\|_{L^{2}}\left(\left\|\nabla n \nabla^{k+1} v\right\|_{L^{2}}\right. \\
& \left.+\left\|\nabla^{2} n \nabla^{k} v\right\|_{L^{2}}+\left\|\nabla^{k+1} n \nabla v\right\|_{L^{2}}\right) \\
\leq & C \delta\left(\|\nabla n\|_{H^{k}}^{2}+\left\|\nabla^{k+1} v\right\|_{L^{2}}^{2}\right) .
\end{aligned}
$$

A similar argument shows that

$$
H_{2}^{2} \leq C \delta\left(\left\|\nabla^{k+1} n\right\|_{L^{2}}^{2}+\|\nabla v\|_{H^{k+1}}^{2}\right) .
$$

By the estimates (36) and (37), we get

$$
H_{2} \leq C \delta\left(\|\nabla n\|_{H^{k}}^{2}+\|\nabla v\|_{H^{k+1}}^{2}\right) .
$$

Similarly, we see that

$$
\begin{gathered}
H_{3} \leq C \delta\|\nabla v\|_{H^{k+1}}^{2}, \\
H_{4} \leq C \delta\left(\|\nabla n\|_{H^{k}}^{2}+\left\|\nabla^{k+1} v\right\|_{L^{2}}^{2}+\|\nabla z\|_{H^{k}}^{2}\right), \\
H_{5} \leq C \delta\left(\|\nabla n\|_{H^{k}}^{2}+\left\|\nabla^{k+1} v\right\|_{L^{2}}^{2}+\|\nabla z\|_{H^{k}}^{2}\right) .
\end{gathered}
$$

Let $H_{6}=H_{6}^{1}+H_{6}^{2}+H_{6}^{3}$. For the first term $H_{6}^{1}$, we exploit the (20), Lemma A.1 and Hölder's inequality to obtain

$$
\begin{aligned}
H_{6}^{1}= & \frac{\hbar^{2}}{4 \gamma_{1}}\left\langle\nabla^{k}\left(\frac{\nabla n \cdot \nabla^{2} n}{(n+1)^{2}}\right), \nabla^{k+2} v\right\rangle \\
\leq & C \hbar^{2} \sum_{0 \leq l \leq k}\left\|\nabla^{l+2} n \nabla^{k-1}\left(\frac{\nabla n}{(n+1)^{2}}\right)\right\|_{L^{2}}\left\|\nabla^{k+2} v\right\|_{L^{2}} \\
\leq & C \hbar^{2}\left(\left\|\nabla^{2} n\right\|_{L^{3}}\left\|\nabla^{k}\left(\frac{\nabla n}{(n+1)^{2}}\right)\right\|_{L^{6}}+\left\|\nabla^{3} n\right\|_{L^{2}}\left\|\nabla^{k-1}\left(\frac{\nabla n}{(n+1)^{2}}\right)\right\|_{L^{\infty}}\right. \\
& \left.+\left\|\nabla^{k+2} n\right\|_{L^{2}}\left\|\frac{\nabla n}{(n+1)^{2}}\right\|_{L^{\infty}}\right)\left\|\nabla^{k+2} v\right\|_{L^{2}} \\
\leq & C \delta \hbar^{2}\left(\left\|\nabla^{2} n\right\|_{H^{k}}^{2}+\left\|\nabla^{k+2} v\right\|_{L^{2}}^{2}\right) .
\end{aligned}
$$

For $H_{6}$, we see that

$$
H_{6} \leq C \delta \hbar^{2}\left(\left\|\nabla^{2} n\right\|_{H^{k}}^{2}+\left\|\nabla^{k+2} v\right\|_{L^{2}}^{2}\right) .
$$

Let $H_{7}=H_{7}^{1}+H_{7}^{2}$. We integrate by parts and use Hölder's inequality to obatin

$$
\begin{aligned}
H_{7}^{2} & =\mu\left\langle\nabla^{k+2} v, \nabla^{k}(g(n) \Delta v)\right\rangle \\
& \leq C \sum_{0 \leq l \leq k}\left\|\nabla^{l} g(n) \nabla^{k+2-l} v\right\|_{L^{2}}\left\|\nabla^{k+2} v\right\|_{L^{2}}
\end{aligned}
$$




$$
\begin{aligned}
\leq & C\left(\|g(n)\|_{L^{\infty}}\left\|\nabla^{k+2} v\right\|_{L^{2}}+\left\|g^{\prime}(n)\right\|_{L^{3}}\left\|\nabla^{k+1} v\right\|_{L^{6}}\right. \\
& \left.+\left\|\nabla^{k} g(n)\right\|\left\|_{L^{6}}\right\| \nabla^{2} v \|_{L^{3}}\right)\left\|\nabla^{k+2} v\right\|_{L^{2}} \\
\leq & C \delta\left(\|\nabla n\|_{H^{k}}^{2}+\left\|\nabla^{k+2} v\right\|_{L^{2}}^{2}\right) .
\end{aligned}
$$

The same estimate holds for $H_{7}^{1}$. Therefore

$$
H_{7} \leq C \delta\left(\|\nabla n\|_{H^{k}}^{2}+\left\|\nabla^{k+2} v\right\|_{L^{2}}^{2}\right) .
$$

As in $H_{8}$, we have

$$
H_{8} \leq C \delta\left(\left\|\nabla^{k+1} n\right\|_{L^{2}}^{2}+\left\|\nabla^{k+1} v\right\|_{L^{2}}^{2}+\|\nabla B\|_{H^{k+1}}^{2}\right) .
$$

In the same manner, it is easy to deduce

$$
\begin{gathered}
H_{9} \leq C \delta\left(\left\|\nabla^{k+1} v\right\|_{L^{2}}^{2}+\|\nabla z\|_{H^{k}}^{2}\right), \\
H_{10} \leq C \delta\left(\|\nabla n\|_{H^{k}}^{2}+\|\nabla v\|_{H^{k}}^{2}+\left\|\nabla^{k+1} z\right\|_{L^{2}}^{2}\right), \\
H_{11} \leq C \delta\left(\|\nabla n\|_{H^{k}}^{2}+\|\nabla z\|_{H^{k+1}}^{2}\right) .
\end{gathered}
$$

Similar to the estimation, we obtain

$$
\begin{aligned}
H_{12}= & \gamma_{3}\left\langle\nabla^{k+1}\left\{f(n)\left[2 \mu|D(v)|^{2}+\lambda(\operatorname{div} u)^{2}\right]\right\}, \nabla^{k+1} z\right\rangle \\
\leq & C\left\langle\nabla^{k}\left[|\nabla v|^{2} f(n)\right], \nabla^{k+2} z\right\rangle \\
= & C \sum_{0 \leq l \leq k} C_{k}^{l}\left\langle\nabla^{l}\left(|\nabla v|^{2}\right) \nabla^{k-1} f(n), \nabla^{k+2} z\right\rangle \\
= & C\left\langle|\nabla v|^{2} \nabla^{k} f(n), \nabla^{k+2} z\right\rangle+C\left\langle\nabla\left(|\nabla v|^{2}\right) \nabla^{k-1} f(n), \nabla^{k+2} z\right\rangle \\
& +C\left\langle\nabla^{k}\left(|\nabla v|^{2}\right) f(n), \nabla^{k+2} z\right\rangle \\
\leq & C\left\|\nabla^{k+2} z\right\|_{L^{2}}\left(\|\nabla v\|_{L^{6}}^{2}\left\|\nabla^{k} f(n)\right\|_{L^{6}}+\|\nabla v\|_{L^{3}}\left\|\nabla^{2} v\right\|_{L^{6}}\left\|\nabla^{k-1} f(n)\right\|_{L^{\infty}}\right. \\
& \left.+\left\|\nabla^{k}\left(|\nabla v|^{2}\right)\right\|\|f(n)\|_{L^{2}}\right) \\
\leq & C \delta\left\|\nabla^{k+2} z\right\|_{L^{2}}\left(\left\|\nabla^{2} v\right\|_{L^{2}}^{2}\left\|\nabla^{k+1} f(n)\right\|_{L^{2}}+\left\|\nabla^{3} v\right\|_{L^{2}}+\left\|\nabla^{k}\left(|\nabla v|^{2}\right)\right\|_{L^{2}}\right) \\
\leq & C \delta\left(\|\nabla n\|_{H^{k}}^{2}+\|\nabla v\|_{H^{k+1}}^{2}+\left\|\nabla^{k+2} z\right\|_{L^{2}}^{2}\right),
\end{aligned}
$$

where

$$
\begin{aligned}
\left\|\nabla^{k}\left(|\nabla v|^{2}\right)\right\|_{L^{2}} & \leq C \sum_{0 \leq I \leq k}\left\|\nabla^{l+1} v \nabla^{k+1-l} v\right\|_{L^{2}} \\
& =C\left\|\nabla v \nabla^{k+1} v\right\|_{L^{2}}+C\left\|\nabla^{2} v \nabla^{k} v\right\|_{L^{2}}+C\left\|\nabla^{k+1} v \nabla v\right\|_{L^{2}} \\
& \leq C\left(2\|\nabla v\|_{L^{3}}\left\|\nabla^{k+1} v\right\|_{L^{6}}+\left\|\nabla^{2} v\right\|_{L^{3}}\left\|\nabla^{k} v\right\|_{L^{6}}\right) \\
& \leq C \delta\|\nabla v\|_{H^{k+1}}^{2} .
\end{aligned}
$$

Similarly, we get 


$$
\begin{aligned}
H_{13}= & \frac{\gamma_{3}}{\gamma_{1}}\left\langle\nabla^{k+1}\left[f(n)|\nabla \times B|^{2}\right], \nabla^{k+1} z\right\rangle \\
\leq & C\left\langle\nabla^{k}\left[|\nabla \times B|^{2} f(n)\right], \nabla^{k+2} z\right\rangle \\
= & C \sum_{0 \leq I \leq k} C_{k}^{l}\left\langle\nabla^{l}\left(|\nabla B|^{2}\right) \nabla^{k-1} f(n), \nabla^{k+2} z\right\rangle \\
= & C\left\langle|\nabla B|^{2} \nabla^{k} f(n), \nabla^{k+2} z\right\rangle+C\left\langle\nabla\left(|\nabla B|^{2}\right) \nabla^{k-1} f(n), \nabla^{k+2} z\right\rangle \\
& +C\left\langle\nabla^{k}\left(|\nabla B|^{2}\right) f(n), \nabla^{k+2} z\right\rangle \\
\leq & C\left\|\nabla^{k+2} z\right\|_{L^{2}}\left(\|\nabla B\|_{L^{6}}^{2}\left\|\nabla^{k} f(n)\right\|_{L^{6}}+\|\nabla B\|_{L^{3}}\left\|\nabla^{2} B\right\|_{L^{6}}\left\|\nabla^{k-1} f(n)\right\|_{L^{\infty}}\right. \\
& \left.+\left\|\nabla^{k}\left(|\nabla B|^{2}\right)\right\|\left\|_{L^{2}}\right\| f(n) \|_{L^{\infty}}\right) \\
\leq & C \delta\left\|\nabla^{k+2} z\right\|_{L^{2}}\left(\left\|\nabla^{2} B\right\|_{L^{2}}^{2}\left\|\nabla^{k+1} f(n)\right\|_{L^{2}}+\left\|\nabla^{3} B\right\|_{L^{2}}+\left\|\nabla^{k}\left(|\nabla B|^{2}\right)\right\|_{L^{2}}\right) \\
\leq & C \delta\left(\|\nabla n\|_{H^{k}}^{2}+\left\|\nabla^{k+2} z\right\|_{L^{2}}^{2}+\|\nabla B\|_{H^{k+1}}^{2}\right) .
\end{aligned}
$$

where

$$
\begin{aligned}
\left\|\nabla^{k}\left(|\nabla B|^{2}\right)\right\|_{L^{2}} & \leq C \sum_{0 \leq I \leq k}\left\|\nabla^{l+1} B \nabla^{k+1-l} B\right\|_{L^{2}} \\
& =C\left\|\nabla B \nabla^{k+1} B\right\|_{L^{2}}+C\left\|\nabla^{2} B \nabla^{k} B\right\|_{L^{2}}+C\left\|\nabla^{k+1} B \nabla B\right\|_{L^{2}} \\
& \leq C\left(2\|\nabla B\|_{L^{3}}\left\|\nabla^{k+1} B\right\|_{L^{6}}+\left\|\nabla^{2} B\right\|_{L^{2}}\left\|\nabla^{k} B\right\|_{L^{6}}\right) \\
& \leq C \delta\|\nabla B\|_{H^{k+1}}^{2} .
\end{aligned}
$$

Similarly, for the terms $H_{14}$ and $H_{15}$, recalling from the estimate of $H_{2}$, we have

$$
\begin{gathered}
H_{14} \leq C \delta\left(\left\|\nabla^{k+1} v\right\|_{L^{2}}^{2}+\|\nabla B\|_{H^{k}}^{2}\right), \\
H_{15} \leq C \delta\left(\|\nabla v\|_{H^{k+1}}^{2}+\left\|\nabla^{k+1} B\right\|_{L^{2}}^{2}\right) .
\end{gathered}
$$

That is to say

$$
(\nabla \times B) \times B=(B \cdot \nabla) B-\frac{1}{2} \nabla\left(|B|^{2}\right),
$$

it follows from (38) that

$$
\begin{aligned}
H_{16} & =-\left\langle\nabla^{k+1}[\nabla \times((\nabla \times B) \times B)], \nabla^{k+1} B\right\rangle \\
& =\left\langle\nabla^{k+1}\left[(B \cdot \nabla) B-\frac{1}{2} \nabla\left(|B|^{2}\right)\right], \nabla^{k+1}(\nabla \times B)\right\rangle \\
& \leq\left\langle\nabla^{k+2}\left(|B|^{2}\right), \nabla^{k+1}(\nabla \times B)\right\rangle+\left\langle\nabla^{k+1}((B \cdot \nabla) B), \nabla^{k+1}(\nabla \times B)\right\rangle
\end{aligned}
$$

For (39), we have

$$
\begin{aligned}
& \left\langle\nabla^{k+1}(\nabla \times B), \nabla^{k+1}((B \cdot \nabla) B)\right\rangle \\
& =\sum_{0 \leq l \leq k+1} C_{k+1}^{l}\left\langle\nabla^{k+2} B, \nabla^{l} B \nabla^{k+2-l} B\right\rangle \\
& =\left\langle\nabla^{k+2} B, B \nabla^{k+2} B\right\rangle+C_{k+1}^{1}\left\langle\nabla^{k+2} B, \nabla B \nabla^{k+1} B\right\rangle
\end{aligned}
$$




$$
\begin{aligned}
& +C_{k+1}^{k}\left\langle\nabla^{k+2} B, \nabla^{k} B \nabla^{2} B\right\rangle+\left\langle\nabla^{k+2} B, \nabla^{k+1} B \nabla B\right\rangle \\
\leq & C\left\|\nabla^{k+2} B\right\|_{L^{2}}\left(\left\|B \nabla^{k+2} B\right\|_{L^{2}}+\left\|\nabla B \nabla^{k+1} B\right\|_{L^{2}}\right. \\
& \left.+\left\|\nabla^{k} B \nabla^{2} B\right\|_{L^{2}}+\left\|\nabla^{k+1} B \nabla B\right\|_{L^{2}}\right) \\
\leq & C \delta\|\nabla B\|_{H^{k+1}}^{2} .
\end{aligned}
$$

Similar to (39), we see

$$
H_{16} \leq C \delta\|\nabla B\|_{H^{k+1}}^{2} .
$$

Consequently, in light of $H_{1} \sim H_{16}$, we get

$$
\begin{aligned}
& \frac{\mathrm{d}}{\mathrm{d} t}\left(\left\|\nabla^{k+1}(n, v, z, B)\right\|_{L^{2}}^{2}+\frac{\hbar^{2}}{4 \gamma_{1}^{2}}\left\|\frac{1}{n+1} \nabla^{k+2} n\right\|_{L^{2}}^{2}\right) \\
& +C\left(\left\|\nabla^{k+2} v\right\|_{L^{2}}^{2}+\left\|\nabla^{k+2} z\right\|_{L^{2}}^{2}+\left\|\nabla^{k+2} B\right\|_{L^{2}}^{2}\right) \\
& \leq C \delta\left(\|\nabla n\|_{H^{k}}^{2}+\hbar^{2}\left\|\nabla^{2} n\right\|_{H^{k}}^{2}+\|\nabla v\|_{H^{k+1}}^{2}+\|\nabla z\|_{H^{k+1}}^{2}+\|\nabla B\|_{H^{k+1}}^{2}\right) .
\end{aligned}
$$

Since $\delta$ is small, (33) is given.

In the following, we consider the energy estimates on the entropy $n$.

Lemma 3.3. It holds that

$$
\begin{aligned}
& \frac{\mathrm{d}}{\mathrm{d} t}\left(\sum_{k=0}^{2}\left\langle\nabla^{k} v, \nabla^{k+1} n\right\rangle+\|\nabla n\|_{H^{2}}^{2}\right)+C\left(\|\nabla n\|_{H^{2}}^{2}+\hbar^{2}\left\|\nabla^{2} n\right\|_{H^{2}}^{2}\right) \\
& \leq C\left(\|\nabla v\|_{H^{2}}^{2}+\|\nabla z\|_{H^{2}}^{2}\right)+C \delta\left(\|\nabla v\|_{H^{3}}^{2}+\|\nabla z\|_{H^{3}}^{2}+\|\nabla B\|_{H^{3}}^{2}\right) .
\end{aligned}
$$

Proof. When $k=0$ to $k=2$, applying $\nabla^{k}$ to the second Equation in (12) and testing by $\nabla^{k+1} n$, we obtain

$$
\begin{aligned}
\gamma_{1} & \left\|\nabla^{k+1} n\right\|_{L^{2}}^{2}+\frac{\hbar^{2}}{4 \gamma_{1}}\left\|\nabla^{k+2} n\right\|_{L^{2}}^{2} \\
= & -\left\langle\nabla^{k} v_{t}, \nabla^{k+1} n\right\rangle+\mu\left\langle\nabla^{k+1} n, \nabla^{k} \Delta v\right\rangle+(\lambda+\mu)\left\langle\nabla^{k+1} n, \nabla^{k+1} \nabla \cdot v\right\rangle \\
& -\left\langle\gamma_{1} \nabla^{k}(v \cdot \nabla v), \nabla^{k+1} n\right\rangle-\left\langle\gamma_{2} \nabla^{k} \nabla z, \nabla^{k+1} n\right\rangle \\
& -\left\langle\nabla^{k}\left(h_{1}(n, z) \nabla n\right), \nabla^{k+1} n\right\rangle-\left\langle\nabla^{k}\left(h_{2}(n, z) \nabla z\right), \nabla^{k+1} n\right\rangle \\
& +\left\langle\frac{\hbar^{2}}{4 \gamma_{1}} \nabla^{k}\left(-g(n) \nabla \Delta n-\frac{\nabla n \cdot \nabla^{2} n}{(n+1)^{2}}-\frac{\nabla n \Delta n}{(n+1)^{2}}+\frac{|\nabla n|^{2} \nabla n}{(n+1)^{3}}\right), \nabla^{k+1} n\right\rangle \\
& -\left\langle\nabla^{k}(g(n)((\mu+\lambda) \nabla \nabla \cdot v)+\mu \Delta v), \nabla^{k+1} n\right\rangle+\left\langle\nabla^{k}\left(\frac{B \cdot \nabla B}{\gamma(n+1)}\right), \nabla^{k+1} n\right\rangle \\
:= & W_{1}+W_{2}+W_{3}+W_{4}+W_{5}+W_{6}+W_{7}+W_{8}+W_{9}+W_{10} \cdot
\end{aligned}
$$

To estimate each term on the right-hand side, we integrate by parts twice, (20) and the continuity equation to deduce

$$
\begin{aligned}
W_{1}= & -\frac{\mathrm{d}}{\mathrm{d} t}\left\langle\nabla^{k} v, \nabla^{k+1} n\right\rangle+\gamma_{1}\left\|\nabla^{k} \nabla \cdot v\right\|_{L^{2}}^{2} \\
& +\gamma_{1}\left\langle\nabla^{k}(\nabla n \cdot v), \nabla^{k} \nabla \cdot v\right\rangle+\gamma_{1}\left\langle\nabla^{k}(n \nabla \cdot v), \nabla^{k} \nabla \cdot v\right\rangle \\
\leq & -\frac{\mathrm{d}}{\mathrm{d} t}\left\langle\nabla^{k} v, \nabla^{k+1} n\right\rangle+\gamma_{1}\left\|\nabla^{k} \nabla \cdot v\right\|_{L^{2}}^{2}
\end{aligned}
$$




$$
\begin{aligned}
& +C\left(\left\|\nabla^{k}(\nabla n \cdot v)\right\|_{L^{2}}+\left\|\nabla^{k}(n \nabla \cdot v)\right\|_{L^{2}}\right)\left\|\nabla^{k+1} v\right\|_{L^{2}} \\
\leq & -\frac{\mathrm{d}}{\mathrm{d} t}\left\langle\nabla^{k} v, \nabla^{k+1} n\right\rangle+\gamma_{1}\left\|\nabla^{k+1} v\right\|_{L^{2}}^{2} \\
& +C\left(\|\nabla n\|_{L^{3}}\left\|\nabla^{k} v\right\|_{L^{6}}+\sum_{1 \leq l \leq k}\left\|\nabla^{l+1} n\right\|_{L^{2}}\left\|\nabla^{k-l} v\right\|_{L^{\infty}}\right. \\
& \left.+\|\nabla \cdot v\|_{L^{3}}\left\|\nabla^{k} n\right\|_{L^{6}}+\sum_{1 \leq l \leq k}\left\|\nabla^{l} \nabla \cdot v\right\|_{L^{2}}\left\|\nabla^{k-l} n\right\|_{L^{\infty}}\right)\left\|\nabla^{k+1} v\right\|_{L^{2}} \\
\leq & -\frac{\mathrm{d}}{\mathrm{d} t}\left\langle\nabla^{k} v, \nabla^{k+1} n\right\rangle+\gamma_{1}\left\|\nabla^{k+1} v\right\|_{L^{2}}^{2}+C \delta\left(\|\nabla n\|_{H^{k}}^{2}+\|\nabla v\|_{H^{k}}^{2}\right) .
\end{aligned}
$$

Similarly, as the estimate of $H_{2}^{1}$, we obtain

$$
\begin{gathered}
W_{2} \leq-\frac{\mu}{2} \frac{\mathrm{d}}{\mathrm{d} t}\left\|\nabla^{k+1} n\right\|_{L^{2}}^{2}+C \delta\left(\|\nabla n\|_{H^{k}}^{2}+\|\nabla v\|_{H^{k+1}}^{2}\right), \\
W_{3} \leq-\frac{\mu+\lambda}{2} \frac{\mathrm{d}}{\mathrm{d} t}\left\|\nabla^{k+1} n\right\|_{L^{2}}^{2}+C \delta\left(\|\nabla n\|_{H^{k}}^{2}+\|\nabla v\|_{H^{k+1}}^{2}\right) .
\end{gathered}
$$

Similar to the estimation on $\mathrm{H}_{2}$, we have

$$
\begin{gathered}
W_{4} \leq C \delta\left(\left\|\nabla^{k+1} n\right\|_{L^{2}}^{2}+\|\nabla v\|_{H^{k}}^{2}\right), \\
W_{5} \leq C\left(\left\|\nabla^{k+1} n\right\|_{L^{2}}^{2}+\|\nabla z\|_{H^{k}}^{2}\right), \\
W_{6} \leq C \delta\|\nabla n\|_{H^{k}}^{2}, \\
W_{7} \leq C \delta\left(\left\|\nabla^{k+1} n\right\|_{L^{2}}^{2}+\|\nabla z\|_{H^{k+1}}^{2}\right) .
\end{gathered}
$$

Let $W_{8}=W_{8}^{1}+W_{8}^{2}+W_{8}^{3}+W_{8}^{4}$. For the terms $W_{8}^{1}$, integrating by parts and Hölder's inequality yields,

$$
\begin{aligned}
W_{8}^{1}= & \frac{\hbar^{2}}{4 \gamma_{1}}\left\langle g^{\prime}(n) \nabla^{k+2} n, \nabla^{k+1} n\right\rangle+\frac{\hbar^{2}}{4 \gamma_{1}}\left\langle g(n) \nabla^{k+2} n, \nabla^{k+2} n\right\rangle \\
& -\frac{\hbar^{2}}{4 \gamma_{1}} \sum_{1 \leq l \leq k} C_{k}^{l}\left\langle\nabla^{l} g(n) \nabla^{k+1-l} \Delta n, \nabla^{k+1} n\right\rangle \\
\leq & C \hbar^{2}\left[\left\|g^{\prime}(n)\right\|_{L^{\infty}}\left\|\nabla^{k+2} n\right\|_{L^{2}}\left\|\nabla^{k+1} n\right\|_{L^{2}}+\|g(n)\|_{L^{\infty}}\left\|\nabla^{k+2} n\right\|_{L^{2}}^{2}\right. \\
& \left.+\left(\left\|g^{\prime}(n)\right\|_{L^{\infty}}\left\|\nabla^{k} \Delta n\right\|_{L^{2}}+\left\|\nabla^{k} g(n)\right\|_{L^{6}}\|\nabla \Delta n\|_{L^{3}}\right)\left\|\nabla^{k+1} n\right\|_{L^{2}}\right] \\
\leq & C \delta \hbar^{2}\left\|\nabla^{2} n\right\|_{H^{k}}^{2} .
\end{aligned}
$$

It is easy to say

$$
W_{8} \leq C \delta \hbar^{2}\left\|\nabla^{2} n\right\|_{H^{k}}^{2} .
$$

Finally, Combing with $H_{7}$ and $H_{8}$, we get

$$
\begin{gathered}
W_{9} \leq C \delta\left(\|\nabla n\|_{H^{k}}^{2}+\|\nabla v\|_{H^{k+1}}^{2}\right), \\
W_{10} \leq C \delta\left(\left\|\nabla^{k+1} n\right\|_{L^{2}}^{2}+\|\nabla B\|_{H^{k+1}}^{2}\right) .
\end{gathered}
$$

Plugging these estimates into (42), we obtain 


$$
\begin{aligned}
& \frac{\mathrm{d}}{\mathrm{d} t}\left(\left\langle\nabla^{k} v, \nabla^{k+1} n\right\rangle+(2 \mu+\lambda)\left\|\nabla^{k+1} n\right\|_{L^{2}}^{2}\right)+C\left(\left\|\nabla^{k+1} n\right\|_{L^{2}}^{2}+\hbar^{2}\left\|\nabla^{k+2} n\right\|_{L^{2}}^{2}\right) \\
& \leq \gamma_{1}\left\|\nabla^{k+1} v\right\|_{L^{2}}^{2}+C\|\nabla z\|_{H^{k}}^{2}+C \delta\left(\|\nabla n\|_{H^{k}}^{2}+\hbar^{2}\left\|\nabla^{2} n\right\|_{H^{k}}^{2}\right. \\
& \left.\quad+\|\nabla v\|_{H^{k+1}}^{2}+\|\nabla z\|_{H^{k+1}}^{2}+\|\nabla B\|_{H^{k+1}}^{2}\right) .
\end{aligned}
$$

Integrating (43) with respect to $t$ and taking $\delta$ sufficiently small, we conclude Lemma 3.3.

Finally, we obtain the global existence.

Proof of Proposition 2.2. Put Lemma 3.1 into Lemma 3.2 and taking $\delta$ is small, we see that

$$
\begin{aligned}
& \frac{\mathrm{d}}{\mathrm{d} t}\left(\|(n, v, z, B)\|_{H^{3}}^{2}+\hbar^{2}\|\nabla n\|_{H^{3}}^{2}\right)+C_{1}\|\nabla v\|_{H^{3}}^{2}+C_{1}\|\nabla z\|_{H^{3}}^{2}+C_{1}\|\nabla B\|_{H^{3}}^{2} \\
& \leq C_{2} \delta\left(\|\nabla n\|_{H^{2}}^{2}+\hbar^{2}\|\nabla n\|_{H^{3}}^{2}+\|\nabla v\|_{L^{2}}^{2}+\|\nabla z\|_{L^{2}}^{2}+\|\nabla B\|_{L^{2}}^{2}\right) .
\end{aligned}
$$

In view of Lemma 3.3, we have

$$
\begin{aligned}
& \frac{\mathrm{d}}{\mathrm{d} t}\left(\sum_{k=0}^{2}\left\langle\nabla^{k} v, \nabla^{k+1} n\right\rangle+\|\nabla n\|_{H^{2}}^{2}\right)+C_{3}\left(\|\nabla n\|_{H^{2}}^{2}+\hbar^{2}\left\|\nabla^{2} n\right\|_{H^{2}}^{2}\right) \\
& \leq C_{4}\left(\|\nabla v\|_{H^{2}}^{2}+\|\nabla z\|_{H^{2}}^{2}\right)+C_{4} \delta\left(\|\nabla v\|_{H^{3}}^{2}+\|\nabla z\|_{H^{3}}^{2}+\|\nabla B\|_{H^{3}}^{2}\right) .
\end{aligned}
$$

Multiplying (45) by $\frac{C_{1} \delta}{C_{4}}$ and adding the result to (44)

$$
\frac{\mathrm{d}}{\mathrm{d} t}\left(\|(n, v, z, B)\|_{H^{3}}^{2}+\hbar^{2}\|\nabla n\|_{H^{3}}^{2}+\sum_{k=0}^{2}\left\langle\nabla^{k} v, \nabla^{k+1} n\right\rangle\right)+C_{5}\|\nabla(v, z, B, \hbar n)\|_{H^{3}}^{2} \leq 0,
$$

where $\delta>0$ is sufficiently small. Consequently, using the fact

$$
\|(n, v, z, B)\|_{H^{3}}^{2}+\hbar^{2}\|\nabla n\|_{H^{3}}^{2}+\sum_{k=0}^{2}\left\langle\nabla^{k} v, \nabla^{k+1} n\right\rangle \sim\|(n, v, z, B)\|_{H^{3}}^{2}+\hbar^{2}\|\nabla n\|_{H^{3}}^{2} .
$$

We show that

$$
\begin{aligned}
& \|(n, v, z, B)\|_{H^{3}}^{2}+\|\hbar \nabla n\|_{H^{3}}^{2}+\int_{0}^{t}\|\nabla(v, z, B, \hbar n)(\tau)\|_{H^{3}}^{2} \mathrm{~d} \tau \\
& \leq C\left(\left\|n_{0}\right\|_{H^{4}}^{2}+\left\|\left(v_{0}, z_{0}, B_{0}\right)\right\|_{H^{3}}^{2}\right),
\end{aligned}
$$

then (46) gives (14).

This completes the whole proof of Propositions 2.2.

\section{Convergence Rates}

In this section, we shall prove the decay rates of the solution stated in Propositions 2.3. To do this, the strategy is to combine all the energy estimated.

We focus on the following homogenous linearized system of (12).

$$
\left\{\begin{array}{l}
n_{t}+\gamma_{1} \nabla \cdot v=0, \\
v_{t}+\gamma_{1} \nabla n+\gamma_{2} \nabla z-\frac{\hbar^{2}}{4 \gamma_{1}} \nabla \Delta n-\mu \Delta v-(\lambda+\mu) \nabla \nabla \cdot v=0, \\
z_{t}-\kappa_{1} \Delta z+\gamma_{2} \nabla \cdot v=0, \\
B_{t}-\Delta B=0 .
\end{array}\right.
$$


Let us denote the matrix-valued differential operator associated with (47) by

$$
\mathbb{A}=\left(\begin{array}{ccc}
0 & \gamma_{1} \nabla \cdot & 0 \\
\gamma_{1} \nabla-\frac{\hbar^{2}}{4 \gamma_{1}} \nabla \Delta & -\mu \Delta-(\lambda+\mu) \nabla \nabla \cdot & \gamma_{2} \nabla \\
0 & \gamma_{2} \nabla . & -\kappa_{1} \Delta
\end{array}\right) .
$$

Hence, we separation of $B$ from $(n, v, z)$. Assume

$$
U(t)=(n(t), v(t) z(t)),
$$

by taking the Fourier transform with respect to the $x$-variable, we have

$$
U(t)=E(t) U(0)=\mathrm{e}^{-t \mathbb{A}} U(0),
$$

$E(t)$ is the solution semigroup defined by $E(t)=\mathrm{e}^{-t \mathbb{A}}, t \geq 0$, cf. [39].

Lemma 4.1. Let $s \geq 0$ be integers. $(n, v, z)$ satisfies the inequalities with the initial data $n_{0} \in H^{s+1} \cap L^{1}, \quad v_{0}, z_{0} \in H^{s} \cap L^{1}$

$$
\begin{gathered}
\|n(t)\|_{L^{2}} \leq C(1+t)^{-\frac{3}{4}}\left(\left\|\left(n_{0}, v_{0}, z_{0}\right)\right\|_{L^{1}}+\left\|\left(n_{0}, v_{0}, z_{0}\right)\right\|_{L^{2}}\right), \\
\left\|\nabla^{k+1} n(t)\right\|_{L^{2}} \leq C(1+t)^{-\frac{3}{4}-\frac{k+1}{2}}\left(\left\|\left(n_{0}, v_{0}, z_{0}\right)\right\|_{L^{1}}+\left\|\left(\nabla^{k+1} n_{0}, \nabla^{k} v_{0}, \nabla^{k} z_{0}\right)\right\|_{L^{2}}\right), \\
\left\|\nabla^{k} v(t)\right\|_{L^{2}} \leq C(1+t)^{-\frac{3}{4}-\frac{k}{2}}\left(\left\|\left(n_{0}, v_{0}, z_{0}\right)\right\|_{L^{1}}+\left\|\left(\nabla^{k+1} n_{0}, \nabla^{k} v_{0}, \nabla^{k} z_{0}\right)\right\|_{L^{2}}\right), \\
\left\|\nabla^{k} z(t)\right\|_{L^{2}} \leq C(1+t)^{-\frac{3}{4}-\frac{k}{2}}\left(\left\|\left(n_{0}, v_{0}, z_{0}\right)\right\|_{L^{1}}+\left\|\left(\nabla^{k+1} n_{0}, \nabla^{k} v_{0}, \nabla^{k} z_{0}\right)\right\|_{L^{2}}\right),
\end{gathered}
$$

where $0 \leq k \leq s$.

Lemma 4.2. Let $r_{1}, r_{2}>0$, then it holds that

$$
\int_{0}^{t}(1+t-\tau)^{-r_{1}}(1+\tau)^{-r_{2}} \mathrm{~d} \tau \leq C\left(r_{1}, r_{2}\right)(1+t)^{-\min \left\{r_{1}, r_{2}, r_{1}+r_{2}-1-\varepsilon\right\}},
$$

for an arbitrarily small $\varepsilon>0$.

To use the $L^{p}-L^{q}$ estimates of the linear problem for the nonlinear system (12) as $N=\left(S_{1}, S_{2}, S_{3}\right)$, then (12) becomes

$$
\begin{aligned}
& U(t)=E(t) U_{0}+\int_{0}^{t} E(t-\tau) N(U(\tau), B(\tau)) \mathrm{d} \tau, \\
& B(t)=F(t) B_{0}+\int_{0}^{t} F(t-\tau) S_{4}(U(\tau), B(\tau)) \mathrm{d} \tau,
\end{aligned}
$$

where $F(t)=\mathrm{e}^{-t \Delta}$. Such that

$$
\left\|F(t) B_{0}\right\|_{L^{p}} \leq C(1+t)^{-\frac{3}{2}\left(\frac{1}{q}-\frac{1}{p}\right)-\frac{k}{2}}\left\|B_{0}\right\|_{L^{q}},
$$

and then

$$
\left\|\nabla^{k} B(t)\right\|_{L^{p}} \leq C(1+t)^{-\frac{3}{2}\left(\frac{1}{q}-\frac{1}{p}\right)-\frac{k}{2}}\left\|B_{0}\right\|_{L^{q}}+C \int_{0}^{t}(1+t-\tau)^{-\frac{3}{2}\left(\frac{1}{q}-\frac{1}{p}\right)-\frac{k}{2}}\left\|S_{4}(\tau)\right\|_{L^{q}} \mathrm{~d} \tau,(51)
$$

Lemma 4.3. We assume $(U, B)$ is a smooth solution

$$
\|\nabla(U, B)(t)\|_{L^{2}} \leq C E_{0}(1+t)^{-\frac{5}{4}}+C \delta \int_{0}^{t}(1+t-\tau)^{-\frac{5}{4}}\|\nabla(U, B)(\tau)\|_{H^{2}} \mathrm{~d} \tau,
$$

where $E_{0}=\left\|n_{0}\right\|_{H^{4} \cap L^{1}}+\left\|\left(v_{0}, z_{0}, B_{0}\right)\right\|_{H^{3} \cap L^{1}}$. 
Proof. We can know

$$
(n, v, z)(t)=\mathrm{e}^{-t \mathbb{A}}\left(n_{0}, v_{0}, z_{0}\right)+\int_{0}^{t} \mathrm{e}^{-(t-\tau) \mathbb{A}}\left(S_{1}, S_{2}, S_{3}\right)(\tau) \mathrm{d} \tau .
$$

It is easy to know,

$$
\begin{aligned}
\|\nabla n(t)\|_{L^{2}} \leq & C(1+t)^{-\frac{5}{4}}\left(\left\|\left(n_{0}, v_{0}, z_{0}\right)\right\|_{L^{1}}+\left\|\left(\nabla n_{0}, v_{0}, z_{0}\right)\right\|_{L^{2}}\right) \\
& +C \int_{0}^{t}(1+t-\tau)^{-\frac{5}{4}}\left(\left\|\left(S_{1}, S_{2}, S_{3}\right)(\tau)\right\|_{L^{1}}+\left\|\left(\nabla S_{1}, S_{2}, S_{3}\right)(\tau)\right\|_{L^{2}}\right) \mathrm{d} \tau \\
\|\nabla v(t)\|_{L^{2}} \leq & C(1+t)^{-\frac{5}{4}}\left(\left\|\left(n_{0}, v_{0}, z_{0}\right)\right\|_{L^{1}}+\left\|\left(\nabla^{2} n_{0}, \nabla v_{0}, \nabla z_{0}\right)\right\|_{L^{2}}\right) \\
+ & C \int_{0}^{t}(1+t-\tau)^{-\frac{5}{4}}\left(\left\|\left(S_{1}, S_{2}, S_{3}\right)(\tau)\right\|_{L^{1}}+\left\|\left(\nabla^{2} S_{1}, \nabla S_{2}, \nabla S_{3}\right)(\tau)\right\|_{L^{2}}\right) \mathrm{d} \tau \\
\|\nabla z(t)\|_{L^{2}} \leq & C(1+t)^{-\frac{5}{4}}\left(\left\|\left(n_{0}, v_{0}, z_{0}\right)\right\|_{L^{1}}+\left\|\left(\nabla^{2} n_{0}, \nabla v_{0}, \nabla z_{0}\right)\right\|_{L^{2}}\right) \\
+ & C \int_{0}^{t}(1+t-\tau)^{-\frac{5}{4}}\left(\left\|\left(S_{1}, S_{2}, S_{3}\right)(\tau)\right\|_{L^{1}}+\left\|\left(\nabla^{2} S_{1}, \nabla S_{2}, \nabla S_{3}\right)(\tau)\right\|_{L^{2}}\right) \mathrm{d} \tau
\end{aligned}
$$

And the nonlinear source terms can be estimated as follows:

$$
\left\|S_{1}\right\|_{L^{1}} \leq C\|\nabla n\|_{L^{2}}\|v\|_{L^{2}}+C\|n\|_{L^{2}}\|\nabla v\|_{L^{2}} \leq C \delta\left(\|\nabla n\|_{L^{2}}+\|\nabla v\|_{L^{2}}\right)
$$

and

$$
\left\|S_{1}\right\|_{H^{1}} \leq C\|\nabla \cdot(n v)\|_{L^{2}}+C\|\nabla \nabla \cdot(n v)\|_{L^{2}} \leq C \delta\|\nabla(n, v)\|_{H^{1}} .
$$

and by Hölder's inequality and Lemma A.1

$$
\begin{aligned}
\left\|\nabla S_{1}\right\|_{H^{1}} & \leq C\|\nabla \nabla \cdot(n v)\|_{L^{2}}+C\left\|\nabla^{2} \nabla \cdot(n v)\right\|_{L^{2}} \\
& \leq C \delta\left(\|\nabla n\|_{H^{2}}+\|\nabla v\|_{H^{2}}\right) .
\end{aligned}
$$

The second term $S_{2}$ is much more complicated, which can be further decomposed into

$$
\begin{aligned}
S_{2}= & -\gamma_{1}(v \cdot \nabla) v-h_{1}(n, z) \nabla n-h_{2}(n, z) \nabla z \\
& +\frac{\hbar^{2}}{4 \gamma_{1}}\left(\frac{|\nabla n|^{2} \nabla n}{(n+1)^{3}}-\frac{\nabla n \Delta n}{(n+1)^{2}}-\frac{\nabla n \cdot \nabla^{2} n}{(n+1)^{2}}-g(n) \nabla \Delta n\right) \\
& -g(n)((\mu+\lambda) \nabla \nabla \cdot v+\mu \Delta v)+\frac{B \cdot \nabla B}{\gamma_{1}(n+1)} \\
:= & S_{21}+S_{22}+S_{23}+S_{24}+S_{25}+S_{26} .
\end{aligned}
$$

The first term $S_{21}$ can be easily bounded by

$$
\left\|S_{21}\right\|_{L^{1}} \leq C\|\nabla v\|_{L^{2}}\|v\|_{L^{2}} \leq C \delta\|\nabla v\|_{L^{2}}
$$

and

$$
\begin{aligned}
\left\|S_{21}\right\|_{H^{1}} & \leq C\|v \cdot \nabla v\|_{L^{2}}+C\|\nabla(v \cdot \nabla v)\|_{L^{2}} \\
& \leq C\|v\|_{L^{\infty}}\|\nabla v\|_{H^{1}}+C\|\nabla v\|_{L^{\infty}}\|\nabla v\|_{L^{2}} \\
& \leq C \delta\|\nabla v\|_{H^{1}} .
\end{aligned}
$$

In a similar way, we get 


$$
\begin{gathered}
\left\|S_{22}\right\|_{L^{1}} \leq C \delta\|\nabla n\|_{L^{2}}, \\
\left\|S_{23}\right\|_{L^{1}} \leq C \delta\|\nabla z\|_{L^{2}}
\end{gathered}
$$

and

$$
\begin{aligned}
\left\|S_{22}\right\|_{H^{1}} & \leq C\left\|h_{1}(n, z) \nabla n\right\|_{L^{2}}+C\left\|\nabla\left(h_{1}(n, z) \nabla n\right)\right\|_{L^{2}} \\
& \leq C \delta\|\nabla n\|_{H^{1}}+C \delta\|\nabla n\|_{L^{2}} \\
& \leq C \delta\|\nabla n\|_{H^{1}}, \\
\left\|S_{23}\right\|_{H^{1}} & \leq C\left\|h_{2}(n, z) \nabla z\right\|_{L^{2}}+C\left\|\nabla\left(h_{2}(n, z) \nabla z\right)\right\|_{L^{2}} \\
& \leq C \delta\|\nabla z\|_{H^{1}}+C \delta\|\nabla z\|_{L^{2}} \\
& \leq C \delta\|\nabla z\|_{H^{1}} .
\end{aligned}
$$

Let $S_{24}=S_{24}^{1}+S_{24}^{2}+S_{24}^{3}+S_{24}^{4}$, we have by Hölder's inequality and Lemma A.1 that

$$
\left\|S_{24}^{4}\right\|_{L^{1}} \leq C\|\nabla g(n)\|_{L^{2}}\|\Delta n\|_{L^{2}} \leq C \delta\|\nabla n\|_{H^{1}}
$$

and

$$
\begin{aligned}
\left\|S_{24}^{4}\right\|_{H^{1}}= & \frac{\hbar^{2}}{4 \gamma_{1}}\|\nabla g(n) \Delta n\|_{H^{1}} \\
\leq & C \hbar^{2}\|\nabla g(n) \Delta n\|_{L^{2}}+C \hbar^{2}\|\nabla(\nabla g(n) \Delta n)\|_{L^{2}} \\
\leq & C \hbar^{2}\|\nabla g(n)\|_{L^{\infty}}\|\Delta n\|_{L^{2}}+C \hbar^{2}\|\nabla g(n)\|_{L^{\infty}}\|\nabla \Delta n\|_{L^{2}} \\
& +C \hbar^{2}\left\|\nabla^{2} g(n)\right\|_{L^{\infty}}\|\Delta n\|_{L^{2}} \\
\leq & C \delta\left(\|\nabla n\|_{H^{1}}+\|\Delta n\|_{H^{1}}\right) \leq C \delta\|\nabla n\|_{H^{2}} .
\end{aligned}
$$

For $S_{24}^{1} \sim S_{24}^{3}$, in a similar way, we have

$$
\left\|S_{24}^{i}\right\|_{L^{1}} \leq C \delta\|\nabla n\|_{H^{1}}, \quad\left\|S_{24}^{i}\right\|_{H^{1}} \leq C \delta\|\nabla n\|_{H^{2}} .
$$

where $i=1,2,3$.

Summing up $S_{24}^{1} \sim S_{24}^{4}$, we obtain

$$
\left\|S_{24}\right\|_{L^{1}} \leq C \delta\|\nabla n\|_{H^{1}}, \quad\left\|S_{24}\right\|_{H^{1}} \leq C \delta\|\nabla n\|_{H^{2}} .
$$

The terms $S_{25}$ and $S_{26}$ can be bounded by

$$
\left\|S_{25}\right\|_{L^{1}} \leq C \delta\|\nabla v\|_{H^{1}}, \quad\left\|S_{26}\right\|_{L^{1}} \leq C \delta\|\nabla B\|_{L^{2}}
$$

and

$$
\left\|F_{25}\right\|_{H^{1}} \leq C \delta\|\nabla v\|_{H^{2}}, \quad\left\|F_{26}\right\|_{H^{1}} \leq C \delta\|\nabla B\|_{H^{2}} .
$$

For $S_{3}$, in a similar way, we have

$$
\begin{aligned}
S_{3}= & -\gamma_{1}(v \cdot \nabla) z-h_{3}(n, z) \nabla \cdot v-\kappa_{1} g(n) \Delta z \\
& +\gamma_{3} f(n)\left[2 \mu|D(v)|^{2}+\lambda(\nabla \cdot v)^{2}\right]+\frac{\gamma_{3}}{\gamma_{1}} f(n)|\nabla \times B|^{2} \\
:= & S_{31}+S_{32}+S_{33}+S_{34}+S_{35} .
\end{aligned}
$$

Similarly, it holds that 


$$
\left\|S_{31}\right\|_{L^{1}} \leq C\|\nabla z\|_{L^{2}}\|v\|_{L^{2}} \leq C \delta\|\nabla z\|_{L^{2}}
$$

and

$$
\begin{aligned}
\left\|S_{31}\right\|_{H^{1}} & \leq C\|v \cdot \nabla z\|_{L^{2}}+C\|\nabla(v \cdot \nabla z)\|_{L^{2}} \\
& \leq C\|v\|_{L^{\infty}}\|\nabla z\|_{H^{1}}+C\|\nabla v\|_{L^{\infty}}\|\nabla z\|_{L^{2}} \\
& \leq C \delta\|\nabla z\|_{H^{1}} .
\end{aligned}
$$

Then using the similar way, we arrive at

$$
\left\|S_{32}\right\|_{L^{1}} \leq C \delta\|\nabla v\|_{L^{2}}
$$

or equivalently,

$$
\begin{aligned}
\left\|S_{32}\right\|_{H^{1}} & \leq C\left\|h_{3}(n, z) \nabla \cdot v\right\|_{L^{2}}+C\left\|\nabla\left(h_{3}(n, z) \nabla \cdot v\right)\right\|_{L^{2}} \\
& \leq C \delta\|\nabla v\|_{H^{1}}+C \delta\|\nabla v\|_{L^{2}} \\
& \leq C \delta\|\nabla v\|_{H^{1}} .
\end{aligned}
$$

In a similar way, we have

$$
\begin{gathered}
\left\|S_{33}\right\|_{L^{1}} \leq C \delta\|\nabla z\|_{H^{1}}, \quad\left\|S_{33}\right\|_{H^{1}} \leq C \delta\|\nabla z\|_{H^{2}}, \\
\left\|S_{34}\right\|_{L^{1}} \leq C \delta\|\nabla v\|_{H^{1}}, \quad\left\|S_{34}\right\|_{H^{1}} \leq C \delta\|\nabla v\|_{H^{2}}, \\
\left\|S_{35}\right\|_{L^{1}} \leq C \delta\|\nabla B\|_{H^{1}}, \quad\left\|S_{35}\right\|_{H^{1}} \leq C \delta\|\nabla B\|_{H^{2}} .
\end{gathered}
$$

Summing these terms, we get

$$
\begin{gathered}
\left\|\left(S_{1}, S_{2}, S_{3}\right)(t)\right\|_{L^{1}} \leq C \delta\left(\|\nabla n\|_{H^{1}}+\|\nabla v\|_{H^{1}}+\|\nabla z\|_{H^{1}}+\|\nabla B\|_{H^{2}}\right), \\
\left\|\nabla S_{1}\right\|_{H^{1}} \leq C \delta\left(\|\nabla n\|_{H^{2}}+\|\nabla v\|_{H^{2}}\right), \\
\left\|\left(S_{1}, S_{2}, S_{3}\right)(t)\right\|_{H^{1}} \leq C \delta\|\nabla(n, v, z, B)\|_{H^{2}} .
\end{gathered}
$$

That is, we obtain

$$
\|\nabla U(t)\|_{L^{2}} \leq C K_{0}(1+t)^{-\frac{5}{4}}+C \delta \int_{0}^{t}(1+t-\tau)^{-\frac{5}{4}}\|\nabla(U, B)(\tau)\|_{H^{2}} \mathrm{~d} \tau,
$$

where $K_{0}=\left\|n_{0}\right\|_{H^{4} \cap L^{1}}+\left\|\left(v_{0}, z_{0}\right)\right\|_{H^{3} \cap L^{1}}$.

The inequality reads that

$$
\begin{aligned}
\|\nabla B(t)\|_{L^{2}} & \leq C(1+t)^{-\frac{5}{4}}\left\|B_{0}\right\|_{L^{1}}+C \int_{0}^{t}(1+t-\tau)^{-\frac{5}{4}}\left\|S_{4}(\tau)\right\|_{L^{1}} \mathrm{~d} \tau \\
& \leq C(1+t)^{-\frac{5}{4}}\left\|B_{0}\right\|_{L^{1}}+C \delta \int_{0}^{t}(1+t-\tau)^{-\frac{5}{4}}\|\nabla(U, B)(\tau)\|_{H^{2}} \mathrm{~d} \tau .
\end{aligned}
$$

Combine (59) and (60), and hence this completes the proof of Lemma 4.3. Now we are in a position to prove Propositions 2.3.

Proof of Proposition 2.3. We do it by two steps.

Step 1. First, for formula

$$
H(t)=\|\nabla(n, v, z, B)\|_{H^{2}}^{2}+\hbar^{2}\left\|\nabla^{2} n\right\|_{H^{2}}^{2}+\sum_{k=1}^{2}\left\langle\nabla^{k} v, \nabla^{k+l} n\right\rangle,
$$

we can assume

$$
H(t) \sim\|\nabla(n, v, z, B)\|_{H^{2}}^{2}+\hbar^{2}\left\|\nabla^{2} n\right\|_{H^{2}}^{2},
$$


then, taking $C_{6}>0$ into

$$
\begin{aligned}
& \frac{1}{C_{6}}\left(\|\nabla(n, v, z, B)\|_{H^{2}}^{2}+\hbar^{2}\left\|\nabla^{2} n\right\|_{H^{2}}^{2}\right) \\
& \leq H(t) \leq C_{6}\left(\|\nabla(n, v, z, B)\|_{H^{2}}^{2}+\hbar^{2}\left\|\nabla^{2} n\right\|_{H^{2}}^{2}\right) .
\end{aligned}
$$

The linear combination of (44) and (45) leads to

$$
\begin{aligned}
& \frac{\mathrm{d} H(t)}{\mathrm{d} t}+C\left(\left\|\nabla^{2}(n, v, z, B)\right\|_{H^{2}}^{2}+\hbar^{2}\left\|\nabla^{2} n\right\|_{H^{2}}^{2}\right) \\
& \leq C \delta\left(\|\nabla(n, v, z, B)\|_{L^{2}}^{2}+\hbar^{2}\|\nabla n\|_{L^{2}}^{2}\right) .
\end{aligned}
$$

Adding $\|\nabla(n, v, z, B)\|_{L^{2}}^{2}+\|\hbar \nabla n\|_{L^{2}}^{2}$ to both sides of the inequality above gives

$$
\frac{\mathrm{d} H(t)}{\mathrm{d} t}+D_{1} H(t) \leq C\|\nabla(U, B)(t)\|_{L^{2}}^{2},
$$

assume that

$$
M(t):=\sup _{0 \leq \tau \leq t}(1+\tau)^{\frac{5}{2}} H(t) .
$$

Notice that $M(t)$ is non-decreasing

$$
\|\nabla(n, v, z, B)\|_{H^{2}}+\left\|\hbar \nabla^{2} n\right\|_{H^{2}} \leq C \sqrt{H(\tau)} \leq C(1+\tau)^{-\frac{5}{4}} \sqrt{M(\tau)}, 0 \leq \tau \leq t \leq T .
$$

Then it follows from Lemma 4.3 that

$$
\begin{aligned}
\|\nabla(U, B)(t)\|_{L^{2}} & \leq C E_{0}(1+t)^{-\frac{5}{4}}+C \delta \int_{0}^{t}(1+t-\tau)^{-\frac{5}{4}}(1+\tau)^{-\frac{5}{4}} \mathrm{~d} \tau \sqrt{M(t)} \\
& \leq C(1+t)^{-\frac{5}{4}}\left(E_{0}+\delta \sqrt{M(t)}\right) .
\end{aligned}
$$

In fact, applying Gronwall's inequality to the Lyapunov-type inequality (61) and using (62), we find that

$$
\begin{aligned}
H(t) & \leq H(0) \mathrm{e}^{-D_{1} t}+C \int_{0}^{t} \mathrm{e}^{-D_{1}(t-\tau)}\|\nabla(U, B)(\tau)\|_{L^{2}}^{2} \mathrm{~d} \tau \\
& \leq H(0) \mathrm{e}^{-D_{1} t}+C \int_{0}^{t}(1+t-\tau)^{-\frac{5}{4}}(1+\tau)^{-\frac{5}{2}} \mathrm{~d} \tau\left(E_{0}+\delta \sqrt{M(t)}\right)^{2} \\
& \leq C(1+t)^{-\frac{5}{2}}\left(H(0)+E_{0}^{2}+\delta^{2} M(t)\right) .
\end{aligned}
$$

In view of (62), we have

$$
M(t) \leq C\left(H(0)+E_{0}^{2}+\delta^{2} M(t)\right)
$$

which implies

$$
M(t) \leq C\left(H(0)+E_{0}^{2}\right) .
$$

Since $\delta>0$ is sufficiently small. Consequently,

$$
\|\nabla(n, v, z, B)\|_{H^{2}}+\left\|\hbar \nabla^{2} n\right\|_{H^{2}} \leq C(1+t)^{-\frac{5}{4}}, \quad 0 \leq t \leq T .
$$

Using (65), we thus get

$$
\|\nabla(n, v, z, B)\|_{L^{2}} \leq C(1+t)^{-\frac{5}{4}},
$$




$$
\begin{gathered}
\|\nabla(n, v, z, B)\|_{H^{2}} \leq C(1+t)^{-\frac{5}{4}}, \\
\left\|\hbar \nabla^{2} n\right\|_{H^{2}} \leq C(1+t)^{-\frac{5}{4}},
\end{gathered}
$$

which also implies from Lemma A.1 that

$$
\|(n, v, z, B)\|_{L^{\infty}} \leq C\|\nabla(n, v, z, B)\|_{H^{1}} \leq C(1+t)^{-\frac{5}{4}} .
$$

Therefore (16), (17) and (18) are obtained. Then

$$
\|(n, v, z, B)\|_{L^{6}} \leq C\|\nabla(n, v, z, B)\|_{L^{2}} \leq C(1+t)^{-\frac{5}{4}} .
$$

Meanwhile via Lemma 4.1 and (48), we have

$$
\begin{aligned}
& \|(n, v, z, B)(t)\|_{L^{2}} \\
& \leq C(1+t)^{-\frac{3}{4}}\left(\left\|\left(n_{0}, v_{0}, z_{0}\right)\right\|_{L^{1}}+\left\|n_{0}\right\|_{H^{1}}+\left\|v_{0}\right\|_{L^{2}}+\left\|z_{0}\right\|_{L^{2}}+\left\|B_{0}\right\|_{L^{1}}\right) \\
& \quad+C \int_{0}^{t}(1+t-\tau)^{-\frac{3}{4}}\left(\left\|\left(S_{1}, S_{2}, S_{3}\right)(\tau)\right\|_{L^{1}}+\left\|S_{1}(\tau)\right\|_{H^{1}}\right. \\
& \left.\quad+\left\|S_{2}(\tau)\right\|_{L^{2}}+\left\|S_{3}(\tau)\right\|_{L^{2}}+\left\|S_{4}(\tau)\right\|_{L^{1}}\right) \mathrm{d} \tau \\
& \leq C E_{0}(1+t)^{-\frac{3}{4}}+C \delta \int_{0}^{t}(1+t-\tau)^{-\frac{3}{4}}\|(\nabla U, \nabla B)(\tau)\|_{H^{2}} \mathrm{~d} \tau \\
& \leq C E_{0}(1+t)^{-\frac{3}{4}}+C \delta\left(H(0)+E_{0}^{2}\right)^{\frac{1}{2}} \int_{0}^{t}(1+t-\tau)^{-\frac{3}{4}}(1+\tau)^{-\frac{5}{4}} \mathrm{~d} \tau \\
& \leq C(1+t)^{-\frac{3}{4}} .
\end{aligned}
$$

Hence, by interpolation, it is easy to see that for any $2 \leq P \leq 6$,

$$
\|(n, v, z, B)(t)\|_{L^{p}} \leq\|(n, v, z, B)(t)\|_{L^{6}}^{\theta}\|(n, v, z, B)(t)\|_{L^{2}}^{1-\theta} \leq C(1+t)^{-\frac{3}{2}\left(1-\frac{1}{p}\right),}
$$

where $\theta=\frac{3(p-2)}{2 p}$, this proves (15).

Step 2. On the other hand, where $0 \leq t \leq T$, we get it by using the estimates above (12), (16) and Lemma A.1.

$$
\begin{aligned}
& \left\|\partial_{t}(n, v, z, B)(t)\right\|_{L^{2}} \\
& \leq C\|\nabla \cdot v\|_{L^{2}}+\left\|S_{1}\right\|_{L^{2}}+C \hbar^{2}\|\nabla \Delta n\|_{L^{2}}+C\|\Delta v\|_{L^{2}} \\
& \quad+C\|\nabla \nabla \cdot v\|_{L^{2}}+C\|\nabla n\|_{L^{2}}+C\|\nabla z\|_{L^{2}}+\left\|S_{2}\right\|_{L^{2}} \\
& \quad+C\|\Delta z\|_{L^{2}}+\left\|S_{3}\right\|_{L^{2}}+\|\Delta B\|_{L^{2}}+\left\|S_{4}\right\|_{L^{2}} \\
& \leq C\|\nabla n\|_{H^{2}}+\|\nabla v\|_{H^{1}}+\|\nabla z\|_{H^{1}}+\|\nabla B\|_{H^{1}} \\
& \leq C(1+t)^{-\frac{5}{4}} .
\end{aligned}
$$

Hence, (19) is proved and we complete the proof of Proposition 2.3.

\section{Conclusion}

Proposition 2.1 gets the local existence, Proposition 2.2 proves a priori estimate, Proposition 2.3 obtains the decay rates of solutions and then Theorem 1.1 is obtained by Propositions 2.1, 2.2 and 2.3. 


\section{Conflicts of Interest}

The author declares no conflicts of interest regarding the publication of this paper.

\section{References}

[1] Wigner, E. (1932) On the Quantum Correction for Thermodynamic Equilibrium. Physical Review Journals Archive, 40, 749-759. https://doi.org/10.1103/PhysRev.40.749

[2] Bohm, D. (1952) A Suggested Interpretation of the Quantum Theory in Terms of Hidden Variables: I. Physical Review Journals Archive, 85, 166-179. https://doi.org/10.1103/PhysRev.85.166

[3] Bohm, D. (1952) A Suggested Interpretation of the Quantum Theory in Terms of Hidden Variables: II. Physical Review Journals Archive, 85, 180-193. https://doi.org/10.1103/PhysRev.85.180

[4] Ancona, M. and Tiersten, H. (1987) Macroscopic Physics of the Silicon Inversion Layer. Physical Review B, 35, 7959-7965. https://doi.org/10.1103/PhysRevB.35.7959

[5] Haas, F. (2011) Quantum Plasmas: An Hydrodynamic Approach. Springer, New York. https://doi.org/10.1007/978-1-4419-8201-8

[6] Pu, X. and Guo, B. (2015) Global Existence and Semiclassical Limit for Quantum Hydrodynamic Equations with Viscosity and Heat Conduction. Kinetic \& Related Models, 9, 165-191. https://doi.org/10.3934/krm.2016.9.165

[7] Pu, X. and Xu, X. (2017) Decay Rates of the Magnetohydrodynamic Model for Quantum Plasmas. Zeitschrift für angewandte Mathematik und Physik, 68, 18. https://doi.org/10.1007/s00033-016-0762-8

[8] Forbes, T. (1991) Magnetic Reconnection in Solar. Geophysical \& Astrophysical Fluid Dynamics, 62, 15-36. https://doi.org/10.1080/03091929108229123

[9] Homann, H. and Grauer, R. (2005) Bifurcation Analysis of Magnetic Reconnection in Hall-MHD Systems. Physica D: Nonlinear Phenomena, 208, 59-72. https://doi.org/10.1016/j.physd.2005.06.003

[10] Shalybkov, D. and Urpin, V. (1997) The Hall Effect and the Decay of Magnetic Fields. Astronomy \& Astrophysics, 321, 685-690.

[11] Balbus, S. and Terquem, C. (2001) Linear Analysis of the Hall Effect in Protostellar Disks. The Astrophysical Journal, 552, 235-247. https://doi.org/10.1086/320452

[12] Wardle, M. (2004) Star Formation and the Hall Effect. Astrophysics and Space Science, 292, 317-323. https://doi.org/10.1023/B:ASTR.0000045033.80068.1f

[13] Mininni, P., Gómez, D. and Mahajan, S. (2003) Dynamo Action in Magnetohydrodynamics and Hall-Magnetohydrodynamics. The Astrophysical Journal, 587, 472-481. https://doi.org/10.1086/368181

[14] Polygiannakis, J. and Moussas, X. (2001) A Review of Magneto-Vorticity Induction in Hall-MHD Plasmas. Plasma Physics and Controlled Fusion, 43, 195-221. https://doi.org/10.1088/0741-3335/43/2/308

[15] Fan, J., Alsaedi, A., Hayat, T., Nakamura, G. and Zhou, Y. (2015) On Strong Solutions to the Compressible Hall-Magnetohydrodynamic System. Nonlinear Analysis. Real World Applications, 22, 423-434. https://doi.org/10.1016/j.nonrwa.2014.10.003

[16] Gao, J. and Yao, Z. (2017) Global Existence and Optimal Decay Rates of Solutions for Compressible Hall-MHD Equations. Discrete \& Continuous Dynamical Sys- 
tems, 36, 3077-3106. https://doi.org/10.3934/dcds.2016.36.3077

[17] Xu, F., Zhang, X., Wu, Y. and Liu, L. (2016) Global Existence and Temporal Decay for the 3D Compressible Hall-Magnetohydrodynamic System. Journal of Mathematical Analysis and Applications, 438, 285-310.

https://doi.org/10.1016/j.jmaa.2016.02.007

[18] Xu, F., Chi, M., Liu, L. and Wu, Y. (2018) Existence, Uniqueness and Optimal Decay Rates for the 3D Compressible Hall-Magnetohydrodynamic System. arXiv:1806.02492.

[19] Chae, D., Degond, P. and Liu, J. (2014) Well-Posedness for Hall-Magneto-hydrodynamics. Annales de I Institut Henri Poincare (C) Non Linear Analysis, 31, 555-565. https://doi.org/10.1016/j.anihpc.2013.04.006

[20] Chae, D. and Lee, J. (2014) On the Blow-Up Criterion and Small Data Global Existence for the Hall-Magnetohydrodynamics. Journal of Differential Equations, 256, 3835-3858. https://doi.org/10.1016/j.jde.2014.03.003

[21] Fan, J., Fukumoto, Y., Nakamura, G. and Zhou, Y. (2015) Regularity Criteria for the Incompressible Hall-MHD System. Zeitschrift fur Angewandte Mathematik und Mechanik, 95, 1156-1160. https://doi.org/10.1002/zamm.201400102

[22] Wan, R. and Zhou, Y. (2015) On Global Existence, Energy Decay and Blow-up Criteria for the Hall-MHD System. Journal of Differential Equations, 259, 5982-6008. https://doi.org/10.1016/j.jde.2015.07.013

[23] Jia, X. and Zhou, Y. (2016) On Regularity Criteria for the 3D Incompressible MHD Equations Involving One Velocity Component. Journal of Mathematical Fluid Mechanics, 18, 187-206. https://doi.org/10.1007/s00021-015-0246-1

[24] Zhao, X. and Zhu, M. (2018) Global Well-Posedness and Asymptotic Behavior of Solutions for the Three-Dimensional MHD Equations with Hall and Ion-Slip Effects. Zeitschrift für angewandte Mathematik und Physik, 69, 22. https://doi.org/10.1007/s00033-018-0907-z

[25] Kawashima, S. (1984) Smooth Global Solutions for Two-Dimensional Equations of Electro-Magneto-Fluid Dynamics. Japan Journal of Applied Mathematics, 1, 207. https://doi.org/10.1007/BF03167869

[26] Umeda, T., Kawashima, S. and Shizuta, Y. (1984) On the Decay of Solutions to the Linearized Equations of Electro-Magneto-Fluid Dynamics. Japan Journal of Applied Mathematics, 1, 435-457. https://doi.org/10.1007/BF03167068

[27] Zhou, Y. (2006) Regularity Criteria for the 3D MHD Equations in Terms of the Pressure. International Journal of Non-Linear Mechanics, 41, 1174-1180. https://doi.org/10.1016/j.ijnonlinmec.2006.12.001

[28] Li, F. and Yu, H. (2011) Optimal Decay Rate of Classical Solutions to the Compressible Magnetohydrodynamic Equations. Proceedings of the Royal Society of Edinburgh Section A: Mathematics, 141, 109-126. https://doi.org/10.1017/S0308210509001632

[29] Tan, Z. and Wang, H. (2013) Optimal Decay Rates of the Compressible Magnetohydrodynamic Equations. Nonlinear Analysis. Real World Applications, 14, 188-201. https://doi.org/10.1016/j.nonrwa.2012.05.012

[30] Fan, J., Jia, X., Nakamura, G. and Zhou, Y. (2015) On Well-Posedness and Blowup Criteria for the Magnetohydrodynamics with the Hall and Ion-Slip Effects. Zeitschrift für angewandte Mathematik und Physik, 66, 1695-1706. https://doi.org/10.1007/s00033-015-0499-9

[31] Hu, X. and Wang, D. (2008) Global Solutions to the Three-Dimensional Full Compressible Magnetohydrodynamic Flows. Communications in Mathematical Physics, 
283, 255-284. https://doi.org/10.1007/s00220-008-0497-2

[32] Bian, D. and Guo, B. (2013) Well-Posedness in Critical Spaces for the Full Compressible MHD Equations. Acta Mathematica Scientia, 33B, 1153-1176. https://doi.org/10.1016/S0252-9602(13)60071-5

[33] Pu, X. and Guo, B. (2013) Global Existence and Convergence Rates of Smooth Solutions for the Full Compressible MHD Equations. Zeitschrift für angewandte Mathematik und Physik, 64, 519-538. https://doi.org/10.1007/s00033-012-0245-5

[34] Gao, J., Tao, Q. and Yao, Z. (2016) Optimal Decay Rates of Classical Solutions for the Full Compressible MHD Equations. Zeitschrift für angewandte Mathematik und Physik, 67, 23. https://doi.org/10.1007/s00033-016-0616-4

[35] He, F., Samet, B. and Zhou, Y. (2018) Boundedness and Time Decay of Solutions to a Full Compressible Hall-MHD System. Bulletin of the Malaysian Mathematical Sciences Society, 41, 2151-2162. https://doi.org/10.1007/s40840-018-0640-y

[36] Donatelli, D. (2003) Local and Global Existence for the Coupled Navier-StokesPoisson Problem. Quarterly of Applied Mathematics, 61, 345-361. https://doi.org/10.1090/qam/1976375

[37] Hu, X. and Wang, D. (2010) Local Strong Solution to the Compressible Viscoelastic Flow with Large Data. Journal of Differential Equations, 249, 1179-1198. https://doi.org/10.1016/j.jde.2010.03.027

[38] Zhang, Y. and Tan, Z. (2007) On the Existence of Solutions to the Navier-StokesPoisson Equations of a Two-Dimensional Compressible Flow. Mathematical Methods in the Applied Sciences, 30, 305-329.

https://doi.org/10.1002/mma.786

[39] Wang, Y. and Tan, Z. (2011) Optimal Decay Rates for the Compressible Fluid Models of Korteweg Type. Journal of Mathematical Analysis and Applications, 379, 256-271. https://doi.org/10.1016/j.jmaa.2011.01.006 


\section{Appendix}

In this appendix, we state some useful inequalities in the Sobolev space.

Lemma A.1. Let $f \in H^{2}\left(\mathbb{R}^{3}\right)$. Then

$$
\begin{gathered}
\|f\|_{L^{\infty}} \leq C\|\nabla f\|_{L^{2}}^{\frac{1}{2}}\|\nabla f\|_{H^{1}}^{\frac{1}{2}} \leq C\|\nabla f\|_{H^{1}}, \\
\|f\|_{L^{6}} \leq C\|\nabla f\|_{L^{2}}, \\
\|f\|_{L^{p}} \leq C\|f\|_{H^{1}}, \quad 2 \leq p \leq 6 .
\end{gathered}
$$

Lemma A.2. Let $f \in W^{m, p}(\Omega)$. Then we get

$$
\|f\|_{C^{0}\left(\mathbb{R}^{3}\right)} \leq C\|f\|_{W^{m, p}(\Omega)} .
$$

Lemma A.3. Let $m \geq 1$ be an integer, then we have

$$
\left\|\nabla^{m}(f g)\right\|_{L^{p}} \leq C\|f\|_{L^{p_{1}}}\left\|\nabla^{m} g\right\|_{L^{p_{2}}}+C\left\|\nabla^{m} f\right\|_{L^{p_{3}^{3}}}\|g\|_{L^{p_{4}}},
$$

and

$$
\left\|\nabla^{m}(f g)-f \nabla^{m} g\right\|_{L^{p}} \leq C\|\nabla f\|_{L^{p_{1}}}\left\|\nabla^{m-1} g\right\|_{L^{p_{2}}}+C\left\|\nabla^{m} f\right\|_{L^{p_{3}}}\|g\|_{L^{p_{4}}},
$$

where $p, p_{1}, p_{2}, p_{3}, p_{4} \in[1, \infty)$ and

$$
\frac{1}{p}=\frac{1}{p_{1}}+\frac{1}{p_{2}}=\frac{1}{p_{3}}+\frac{1}{p_{4}} .
$$

